Article

\title{
Push-Pull Chromophores Based on the Naphthalene Scaffold: Potential Candidates for Optoelectronic Applications
}

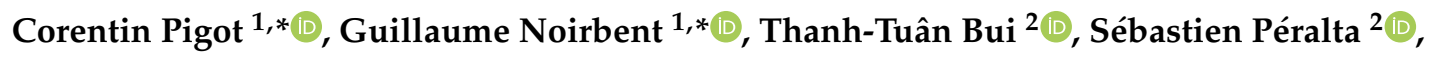 \\ Didier Gigmes ${ }^{1}$, Malek Nechab ${ }^{1}$ and Frédéric Dumur ${ }^{1, *(D)}$ \\ 1 Aix Marseille Univ, CNRS, ICR UMR7273, F-13397 Marseille, France; didier.gigmes@univ-amu.fr (D.G.); \\ malek.nechab@univ-amu.fr (M.N.) \\ 2 Laboratoire de Physicochimie des Polymères et des Interfaces (LPPI), Université de Cergy Pontoise, \\ 5 mail Gay Lussac, F-95000 Neuville-sur-Oise, France; tbui@u-cergy.fr (T.-T.B.); \\ sebastien.Peralta@u-cergy.fr (S.P.) \\ * Correspondence: corentin.pigot@univ-amu.fr (C.P.); Guillaume.noirbent@outlook.fr (G.N.); \\ frederic.dumur@univ-amu.fr (F.D.)
}

Received: 5 April 2019; Accepted: 22 April 2019; Published: 24 April 2019

\begin{abstract}
A series of ten push-pull chromophores comprising $1 H$-cyclopenta[ $b]$ naphthalene$1,3(2 \mathrm{H})$-dione as the electron-withdrawing group have been designed, synthesized, and characterized by UV-visible absorption and fluorescence spectroscopy, cyclic voltammetry and theoretical calculations. The solvatochromic behavior of the different dyes has been examined in 23 solvents and a positive solvatochromism has been found for all dyes using the Kamlet-Taft solvatochromic relationship, demonstrating the polar form to be stabilized in polar solvents. To establish the interest of this polyaromatic electron acceptor only synthesizable in a multistep procedure, a comparison with the analog series based on the benchmark indane-1,3-dione ( $1 H$-indene-1,3(2H)-dione) has been done. A significant red-shift of the intramolecular charge transfer band has been found for all dyes, at a comparable electron-donating group. Parallel to the examination of the photophysical properties of the different chromophores, a major improvement of the synthetic procedure giving access to $1 H$-cyclopenta[ $b]$ naphthalene-1,3(2H)-dione has been achieved.
\end{abstract}

Keywords: push-pull dyes; chromophore; naphthalene; solvatochromism

\section{Introduction}

During the past decades, push-pull molecules have attracted much attention due to their numerous applications ranging from non-linear optical (NLO) applications [1,2] to organic photovoltaics (OPVs) [3,4], organic field effect transistors (OFETs) [5], organic light-emitting diodes (OLEDs) [6], photorefractive applications [7], colorimetric pH sensors [8], ions detection [9], biosensors [10], gas sensors [11], or photoinitiators of polymerization [12-16]. Typically, push-pull chromophores are based on an electron donor and an electron acceptor connected to each other by mean of a saturated or a conjugated spacer [17]. As the first manifestation of the mutual interaction between the two partners, a broad absorption band corresponding to the intramolecular charge transfer (ICT) interaction can be detected in the visible to the near-infrared region. Position of this absorption band typically depends on the electron-donating ability of the donor and the electron-releasing ability of the acceptor, and a bathochromic shift of this transition is observed upon improvement of the strength of the donor and/or the acceptor. This ICT band can even be detected in the near and far infrared region for electron-acceptors based on poly(nitrofluorenes) [18]. Among electron acceptors, indane-1,3-dione (1H-indene-1,3(2H)-dione) EA1 has been extensively studied due to its commercial 
availability, its low cost and the possibility to design push-pull dyes by a mean of one of the simplest reaction of Organic Chemistry, namely the Knoevenagel reaction [19-23]. By chemical engineering, its electron-withdrawing ability can be drastically improved by condensation of one or two malononitrile units under basic conditions, furnishing 2-(3-oxo-2,3-dihydro- $1 \mathrm{H}$-inden-1-ylidene)malononitrile EA2 [24] and 2,2'-(1H-indene-1,3 (2H)-diylidene)dimalononitrile EA3 [25] (see Figure 1). Based on the strong electron-withdrawing abilities of EA2 and EA3, push-pull dyes with non-fullerene acceptors and small energy gaps have been developed [26,27]. As a possible alternative to improve the electron-accepting ability, extension of the aromaticity of the acceptor can be envisioned. Using this strategy, a red-shift of the ICT band combined with an enhancement of the molar extinction coefficient can be both obtained for these new push-pull derivatives, relative to that obtained with the parent electron acceptor.

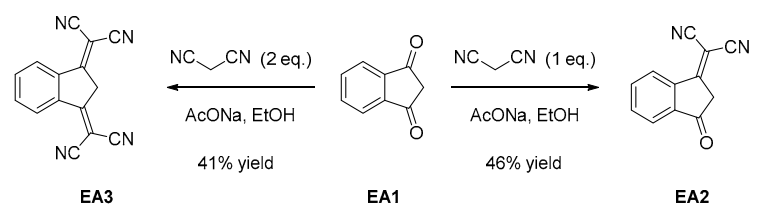

Figure 1. Electron acceptors EA1-EA3.

In this article, an extended version of the well-known indane-1,3-dione, i.e., $1 H$-cyclopenta[b] naphthalene-1,3(2H)-dione EA4, has been used for the design of ten push-pull dyes. It has to be noticed that even if the synthesis of EA4 is reported in the literature since 2006 [28], only one report mentions the development of anion sensors with EA4 in 2013 [29] and the first push-pull dyes have been developed in 2017 for photovoltaics applications [30,31]. Considering the scarcity of studies devoted to this electron acceptor, a series of 10 push-pull chromophores PP1-PP10 have been developed with EA4. To evidence the contribution of the additional aromatic ring in EA4 relative to that of EA1, 10 dyes PP11-PP20 comprising EA1 as the electron acceptor have been synthesized for comparison (see Figure 2).

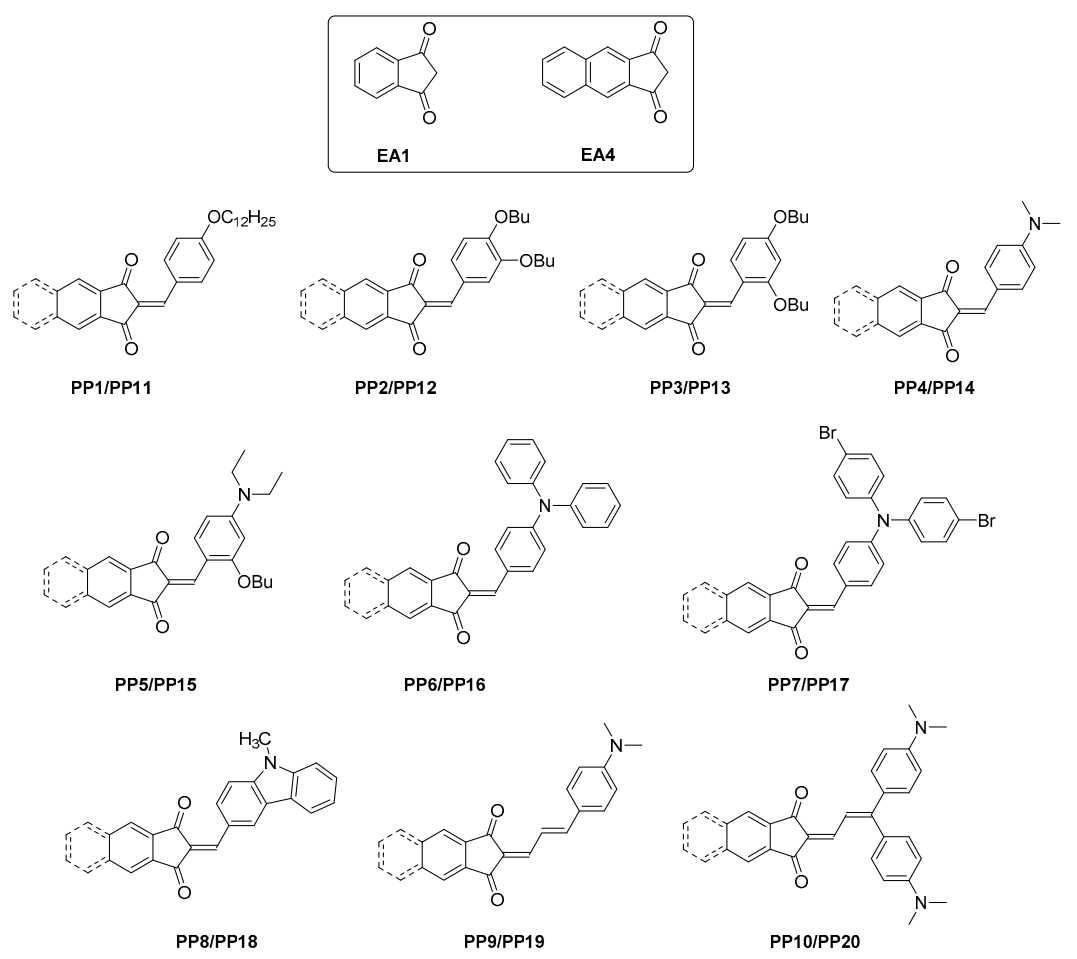

Figure 2. Chemical structures of the 20 dyes PP1-PP20 examined in this study. PP1-PP10 were made from EA4, while PP11-PP20 were made from EA1. 
The photophysical properties of the series of 20 dyes as well as their electrochemical properties have been investigated. To support the experimental results, theoretical calculations have been carried out. Finally, the solvatochromic properties have also been examined.

\section{Materials and Methods}

All reagents and solvents were purchased from Aldrich, Alfa Aesar or TCI Europe and used as received without further purification. Mass spectroscopy was performed by the Spectropole of Aix-Marseille University. Electron spray ionization (ESI) mass spectral analyses were recorded with a 3200 QTRAP (Applied Biosystems SCIEX) mass spectrometer. The HRMS mass spectral analysis was performed with a QStar Elite (Applied Biosystems SCIEX) mass spectrometer. Elemental analyses were recorded with a Thermo Finnigan EA 1112 elemental analysis apparatus driven by the Eager 300 software. ${ }^{1} \mathrm{H}$ and ${ }^{13} \mathrm{C}$ nuclear magnetic resonance (NMR) spectra were determined at room temperature in $5 \mathrm{~mm}$ outer diameter (o.d.) tubes on a Bruker Avance 400 spectrometer of the Spectropole: ${ }^{1} \mathrm{H}(400 \mathrm{MHz})$ and ${ }^{13} \mathrm{C}(100 \mathrm{MHz})$. The ${ }^{1} \mathrm{H}$ chemical shifts were referenced to the solvent peak $\mathrm{CDCl}_{3}(7.26 \mathrm{ppm})$ and the ${ }^{13} \mathrm{C}$ chemical shifts were referenced to the solvent peak $\mathrm{CDCl}_{3}(77 \mathrm{ppm})$. UV-visible absorption spectra were recorded on a Varian Cary 50 Scan UV Visible Spectrophotometer, with concentration of $5 \times 10^{-3} \mathrm{M}$, corresponding to diluted solutions. Fluorescence spectra were recorded using a Jasco FP 6200 spectrometer. The electrochemical properties of the investigated compounds were measured in acetonitrile by cyclic voltammetry, scan rate $100 \mathrm{mV} \cdot \mathrm{s}^{-1}$, with tetrabutylammonium tetrafluoroborate $(0.1 \mathrm{M})$ as a supporting electrolyte in a standard one-compartment, three-electrode electrochemical cell under an argon stream using a VSP BioLogic potentiostat. The working, pseudo-reference and counter electrodes were platinum disk $(\varnothing=1 \mathrm{~mm}), \mathrm{Ag}$ wire, and Au wire gauze, respectively. Ferrocene was used as an internal standard, and the potentials are referred to the reversible formal potential of this compound. Computational details: All quantum mechanical calculations were computed using Gaussian Package [32]. All geometry optimizations were performed using the density functional theory (DFT) with the global hybrid exchange-correlation functional B3LYP [33] and all minima on the potential energy surface were verified via a calculation of vibrational frequencies, ensuring no imaginary frequencies were present. The Pople double-zeta basis set with a double set of polarization functions on non-hydrogen atoms $(6-3111 \mathrm{G}(\mathrm{d}, \mathrm{p}))[34,35]$ was used throughout. This computational approach was chosen in consistency with previous works, as it provides good agreement with experimental data. Excited states were probed using the time dependent density functional theory (TD-DFT) using the same function. All transitions (singlet-singlet) were calculated vertically with respect to the singlet ground state geometry. Solvent effects were taken into account by using the implicit polarizable continuum model (PCM) [36,37]. Dichloromethane (DCM) where chosen in analogy with the experiments. Computed spectra were simulated by convoluting each transition with Gaussians functions-centered on each absorption maximum using a constant full width at half maximum (FWHM) value of $0.2 \mathrm{eV}$. The assignment of electronic transitions for $\lambda_{\max }$ has been determined with GaussSum 3.0 software [38]. 4-(Dodecyloxy)benzaldehyde D1 [39], 3,4-dibutoxy-benzaldehyde D2 [40], 2,4-dibutoxy-benzaldehyde D3 [41], 4-(diphenylamino)benzaldehyde D6 [42], 4-(bis(4-bromophenyl)-amino)benzaldehyde D7 [43], and 9-methyl-9H-carbazole-3-carbaldehyde D8 [44], 3-(4-(dimethylamino)phenyl)acrylaldehyde D9 [45], and 3,3-bis(4-(dimethylamino)phenyl)-acrylaldehyde) D10 [46] were synthesized, as previously reported, without modifications and in similar yields.

\subsection{Synthesis of the Dyes}

\subsubsection{2-Butoxy-4-(Diethylamino)Benzaldehyde D5}

A mixture of 4-(diethylamino)salicylaldehyde ( $26.5 \mathrm{~g}, 137 \mathrm{mmol}, \mathrm{M}=193.24 \mathrm{~g} / \mathrm{mol}), 1$-bromobutane $(21.8 \mathrm{~g}, 159 \mathrm{mmol})$ and potassium carbonate $(18.9 \mathrm{~g}, 152.2 \mathrm{mmol})$ were heated in $N, N$-dimethylformamide (DMF) $(250 \mathrm{~mL})$ at reflux overnight. The solution was concentrated before addition of water. Extraction of the product was carried out with diethyl ether. The ethereal solution was washed with several 
portions of water to remove remaining DMF, dried over $\mathrm{MgSO}_{4}$ and concentrated to yield the product as an orange oil $\left(32.1 \mathrm{~g}, 128.73 \mathrm{mmol}, 94 \%\right.$ yield). ${ }^{1} \mathrm{H} \mathrm{nmR}\left(\mathrm{CDCl}_{3}\right) \delta: 0.91(\mathrm{t}, 3 \mathrm{H}, \mathrm{J}=7.4 \mathrm{~Hz}), 1.14(\mathrm{t}, 6 \mathrm{H}$, $\mathrm{J}=7.1 \mathrm{~Hz}), 1.42-1.48(\mathrm{~m}, 2 \mathrm{H}), 1.71-1.78(\mathrm{~m}, 2 \mathrm{H}), 3.34(\mathrm{q}, 4 \mathrm{H}, \mathrm{J}=7.1 \mathrm{~Hz}), 3.96(\mathrm{t}, 2 \mathrm{H}, \mathrm{J}=6.3 \mathrm{~Hz}), 5.95$ $(\mathrm{d}, 1 \mathrm{H}, \mathrm{J}=2.3 \mathrm{~Hz}), 6.19(\mathrm{dd}, 1 \mathrm{H}, \mathrm{J}=9.0 \mathrm{~Hz}, \mathrm{~J}=2.3 \mathrm{~Hz}), 7.63$ (d, 1H, J = 9.0 Hz), 10.1 (s, 1H, CHO); ${ }^{13} \mathrm{C} \mathrm{nmR}\left(\mathrm{CDCl}_{3}\right)$ ) $: 12.6,13.8,19.3,31.2,44.7,67.8,93.2,104.2,114.4,130.1,153.8,163.9$, and 187.1; HRMS (ESI MS) m/z: theor: 249.1729 found: 249.1733 ([M] ${ }^{+\cdot}$ detected).

\subsection{2. $1 H$-Cyclopenta[b]Naphthalene-1,3(2H)-Dione EA4}

Diethyl naphthalene-2,3-dicarboxylate $(10 \mathrm{~g}, 38.5 \mathrm{mmol}, 1$ eq., $\mathrm{M}=272.30 \mathrm{~g} / \mathrm{mol})$ was suspended in extra dry EtOAc $(24 \mathrm{~mL})$ and $\mathrm{NaH} 95 \%$ in oil $(2.44 \mathrm{~g}, 96.4 \mathrm{mmol}, 2.5 \mathrm{eq}$.$) was added. The reaction$ media was refluxed at $105^{\circ} \mathrm{C}$ for $5 \mathrm{~h}$. After cooling, the yellow solid was filtered off and thoroughly washed with a mixture of EtOH-Et ${ }_{2} \mathrm{O} 50 / 50$. Treatment of this solid with $200 \mathrm{~mL}$ of a $1 \mathrm{M} \mathrm{HCl}$ solution under reflux for $1.5 \mathrm{~h}$ furnished a new solid. After cooling, the solid was filtered off, washed with water and recrystallized in toluene $(200 \mathrm{~mL})$. The product was obtained as a brown solid $(6.9 \mathrm{~g}, 35.27 \mathrm{mmol}$, 91\% yield). ${ }^{1} \mathrm{H} \mathrm{nmR}\left(\mathrm{CDCl}_{3}\right) \delta: 3.38(\mathrm{~s}, 2 \mathrm{H}), 7.66-7.75(\mathrm{~m}, 2 \mathrm{H}), 8.10-8.13(\mathrm{~m}, 2 \mathrm{H}), 8.52(\mathrm{~s}, 2 \mathrm{H}) ;{ }^{13} \mathrm{C} \mathrm{nmR}$ $\left(\mathrm{CDCl}_{3}\right) \delta: 46.7,124.3,129.7,130.6,136.4,138.2$, and 197.6; HRMS (ESI MS) $\mathrm{m} / \mathrm{z}$ : theor: 196.0524 found: $196.0526\left([\mathrm{M}]^{+} \cdot\right.$ detected).

\subsection{General Procedure for the Synthesis of PP1-PP10:}

$1 H$-Cyclopenta[b]naphthalene-1,3(2H)-dione EA4 $(0.5 \mathrm{~g}, 2.55 \mathrm{mmol})$ and the appropriate substituted benzaldehyde $(2.55 \mathrm{mmol}, 1$. eq.) were dissolved in absolute ethanol $(50 \mathrm{~mL})$ and a few drops of piperidine were added. The reaction mixture was refluxed and progress of the reaction was followed by thin layer chromatography (TLC). After cooling, a precipitate formed. It was filtered off, washed several times with ethanol and dried under vacuum.

\subsubsection{2-(4-(Dodecyloxy)Benzylidene)-1H-Cyclopenta[b]Naphthalene-1,3(2H)-Dione PP1}

4-(Dodecyloxy)benzaldehyde $(0.74 \mathrm{~g}, 2.55 \mathrm{mmol}), \mathrm{m}_{\exp }=1.05 \mathrm{~g}, 2.24 \mathrm{mmol}, 88 \%$ yield. ${ }^{1} \mathrm{H} \mathrm{nmR}$ $\left(\mathrm{CDCl}_{3}\right) \delta: 0.88(\mathrm{t}, 3 \mathrm{H}, \mathrm{J}=6.2 \mathrm{~Hz}), 1.27-1.40(\mathrm{~m}, 16 \mathrm{H}), 1.46(\mathrm{qt}, 2 \mathrm{H}, \mathrm{J}=7.5 \mathrm{~Hz}), 1.83(\mathrm{qt}, 2 \mathrm{H}, \mathrm{J}=6.7$ $\mathrm{Hz}), 4.09(\mathrm{t}, 2 \mathrm{H}, \mathrm{J}=6.5 \mathrm{~Hz}), 7.02(\mathrm{~d}, 2 \mathrm{H}, \mathrm{J}=8.7 \mathrm{~Hz}), 7.67-7.69(\mathrm{~m}, 2 H), 7.94(\mathrm{~s}, 1 H), 8.09-8.10(\mathrm{~m}$, $2 H), 8.49(\mathrm{~d}, 1 \mathrm{H}, \mathrm{J}=3.0 \mathrm{~Hz}), 8.62(\mathrm{~d}, 2 \mathrm{H}, \mathrm{J}=8.7 \mathrm{~Hz}) ;{ }^{13} \mathrm{C} \mathrm{nmR}\left(\mathrm{CDCl}_{3}\right)$ 8: 14.1, 22.7, 26.0, 29.1, 29.3, 29.55, 29.59, 29.63, 29.66, 31.92, 114.9, 123.8, 123.9, 126.5, 128.2, 129.0, 129.1, 130.4, 130.5, 135.6, 136.3, 136.5, 137.6, 137.7, 148.0, 164.2, 189.4, and 190.7; HRMS (ESI MS) m/z: theor: 468.2664 found: 468.2665 $\left([\mathrm{M}]^{+} \cdot\right.$ detected).

\subsubsection{2-(3,4-Dibutoxybenzylidene)-1H-Cyclopenta[b]Naphthalene-1,3(2H)-Dione PP2}

3,4-Dibutoxybenzaldehyde $(0.64 \mathrm{~g}, 2.55 \mathrm{mmol}), \mathrm{m}_{\exp }=0.92 \mathrm{~g}, 2.15 \mathrm{mmol}, 84 \%$ yield. ${ }^{1} \mathrm{H} \mathrm{nmR}$ $\left(\mathrm{CDCl}_{3}\right) \delta: 1.01(\mathrm{t}, 3 \mathrm{H}, \mathrm{J}=7.6 \mathrm{~Hz}), 1.04(\mathrm{t}, 3 \mathrm{H}, \mathrm{J}=7.5 \mathrm{~Hz}), 1.50-1.64(\mathrm{~m}, 4 \mathrm{H}), 1.82-1.94(\mathrm{~m}, 4 \mathrm{H}), 4.14$ $(\mathrm{t}, 2 \mathrm{H}, \mathrm{J}=6.5 \mathrm{~Hz}), 4.26(\mathrm{t}, 2 \mathrm{H}, \mathrm{J}=6.4 \mathrm{~Hz}), 6.97(\mathrm{~d}, 1 \mathrm{H}, \mathrm{J}=8.4 \mathrm{~Hz}), 7.66-7.69(\mathrm{~m}, 2 \mathrm{H}), 7.85(\mathrm{~d}, 1 \mathrm{H}, \mathrm{J}=7.1$ $\mathrm{Hz}), 7.92(\mathrm{~s}, 1 H), 8.07-8.10(\mathrm{~m}, 2 H), 8.49(\mathrm{~s}, 2 H), 8.83(\mathrm{~s}, 1 H) ;{ }^{13} \mathrm{C} \mathrm{nmR}\left(\mathrm{CDCl}_{3}\right) \delta: 13.8,13.9,19.2,19.3$, 31.0, 31.2, 68.8, 68.9, 112.1, 117.8, 123.8, 123.9, 126.9, 128.0, 129.0, 129.1, 130.4, 130.5, 131.7, 135.6, 136.3, 136.5, 137.6, 148.7, 148.8, 154.7, 189.5, and 190.7; HRMS (ESI MS) m/z: theor: 428.1988 found: 428.1987 $\left([\mathrm{M}]^{+} \cdot\right.$ detected).

\subsubsection{2-(2,4-Dibutoxybenzylidene)-1H-Cyclopenta[b]Naphthalene-1,3(2H)-Dione PP3}

2,4-Dibutoxybenzaldehyde (0.64 g, $2.55 \mathrm{mmol}), \mathrm{m}_{\exp }=0.95 \mathrm{~g}, 2.22 \mathrm{mmol}, 87 \%$ yield. ${ }^{1} \mathrm{H} \mathrm{nmR}$ $\left(\mathrm{CDCl}_{3}\right) \delta: 1.00(\mathrm{t}, 3 \mathrm{H}, \mathrm{J}=7.4 \mathrm{~Hz}), 1.04(\mathrm{t}, 3 \mathrm{H}, \mathrm{J}=7.4 \mathrm{~Hz}), 1.49-1.63(\mathrm{~m}, 4 \mathrm{H}), 1.82(\mathrm{qt}, 2 \mathrm{H}, \mathrm{J}=7.7 \mathrm{~Hz})$, $1.91(\mathrm{qt}, 2 \mathrm{H}, \mathrm{J}=6.4 \mathrm{~Hz}), 4.09(\mathrm{t}, 4 \mathrm{H}, \mathrm{J}=6.2 \mathrm{~Hz}), 6.44(\mathrm{~d}, 1 \mathrm{H}, \mathrm{J}=0.9 \mathrm{~Hz}), 6.64(\mathrm{dd}, 1 \mathrm{H}, \mathrm{J}=11.0 \mathrm{~Hz}, \mathrm{~J}=0.9$ $\mathrm{Hz}), 7.64-7.67(\mathrm{~m}, 2 \mathrm{H}), 8.06-8.09(\mathrm{~m}, 2 \mathrm{H}), 8.44(\mathrm{~s}, 2 \mathrm{H}), 8.62(\mathrm{~s}, 1 \mathrm{H}), 9.37(\mathrm{~d}, 1 \mathrm{H}, \mathrm{J}=9.0 \mathrm{~Hz}) ;{ }^{13} \mathrm{C} \mathrm{nmR}$ $\left(\mathrm{CDCl}_{3}\right)$ ): 13.77, 13.83, 19.17, 19.32, 31.04-31.13, 68.2, 68.7, 98.8, 106.2, 116.5, 123.44, 123.48, 127.1, 128.7, 
128.8, 130.3, 130.4, 135.8, 136.3, 136.4, 136.9, 137.7, 142.4, 163.1, 166.6, 189.5, and 191.0; HRMS (ESI MS) $\mathrm{m} / \mathrm{z}$ : theor: 428.1988 found: $428.1986\left([\mathrm{M}]^{+}\right.$detected).

\subsubsection{2-(4-(Dimethylamino)Benzylidene)-1H-Cyclopenta[b]Naphthalene-1,3(2H)-Dione PP4}

4-Dimethylaminobenzaldehyde $(0.38 \mathrm{~g}, 2.55 \mathrm{mmol}), \mathrm{m}_{\exp }=0.62 \mathrm{~g}, 1.89 \mathrm{mmol}, 74 \%$ yield. ${ }^{1} \mathrm{H} \mathrm{nmR}$ $\left(\mathrm{CDCl}_{3}\right) \delta: 3.16(\mathrm{~s}, 6 \mathrm{H}), 6.74(\mathrm{~d}, 2 \mathrm{H}, \mathrm{J}=9.0 \mathrm{~Hz}), 7.62-7.64(\mathrm{~m}, 2 \mathrm{H}), 7.87(\mathrm{~s}, 1 H), 8.05-8.06(\mathrm{~m}, 2 \mathrm{H}), 8.39(\mathrm{~d}$, $2 \mathrm{H}, \mathrm{J}=2.6 \mathrm{~Hz}), 8.61(\mathrm{~d}, 2 \mathrm{H}, \mathrm{J}=8.6 \mathrm{~Hz}) ;{ }^{13} \mathrm{C} \mathrm{nmR}\left(\mathrm{CDCl}_{3}\right) \delta: 40.1,111.5,122.4,122.9,123.1,125.1,128.5$, 128.6, 130.2, 130.3, 135.9, 136.1, 136.3, 137.8, 138.5, 148.5, 154.3, 189.6, and 191.4; HRMS (ESI MS) m/z: theor: 327.1259 found: $327.1260\left([\mathrm{M}]^{+}\right.$. detected).

\subsubsection{2-(2-Butoxy-4-(Diethylamino)Benzylidene)-1H-Cyclopenta[b]Naphthalene-1,3(2H)-Dione PP5}

2-Butoxy-4-(diethylamino)benzaldehyde ( $0.63 \mathrm{~g}, 2.55 \mathrm{mmol}), \mathrm{m}_{\exp }=1.02 \mathrm{~g}, 2.38 \mathrm{mmol}, 94 \%$ yield. ${ }^{1} \mathrm{H} \mathrm{nmR}\left(\mathrm{CDCl}_{3}\right) \delta: 1.04(\mathrm{t}, 3 \mathrm{H}, \mathrm{J}=7.4 \mathrm{~Hz}), 1.28(\mathrm{t}, 6 \mathrm{H}, \mathrm{J}=7.1 \mathrm{~Hz}), 1.60(\mathrm{qt}, 2 \mathrm{H}, \mathrm{J}=7.4 \mathrm{~Hz}), 1.92(\mathrm{qt}, 2 \mathrm{H}, \mathrm{J}$ $=7.8 \mathrm{~Hz}), 3.50(\mathrm{q}, 4 \mathrm{H}, \mathrm{J}=7.1 \mathrm{~Hz}), 4.08(\mathrm{t}, 2 \mathrm{H}, \mathrm{J}=6.4 \mathrm{~Hz}), 6.03(\mathrm{~d}, 1 \mathrm{H}, \mathrm{J}=2.0 \mathrm{~Hz}), 6.43(\mathrm{dd}, 1 \mathrm{H}, \mathrm{J}=9.3$ $\mathrm{Hz}, \mathrm{J}=2.0 \mathrm{~Hz}), 7.59-7.62(\mathrm{~m}, 2 \mathrm{H}), 8.03-8.05(\mathrm{~m}, 2 \mathrm{H}), 8.34(\mathrm{~s}, 2 H), 8.58(\mathrm{~s}, 1 H), 9.50(\mathrm{~d}, 1 \mathrm{H}, \mathrm{J}=9.3 \mathrm{~Hz})$; ${ }^{13} \mathrm{C} \mathrm{nmR}\left(\mathrm{CDCl}_{3}\right)$ 8: 12.8, 13.9, 19.4, 31.1, 45.1, 68.2, 93.1, 105.1, 113.2, 122.37, 122.40, 123.1, 128.1, 128.2, 130.1, 130.2, 136.08, 136.11, 136.3, 137.8, 138.0, 155.2, 164.1, 189.8, and 191.9; HRMS (ESI MS) m/z: theor: 427.2147 found: $427.2149\left([\mathrm{M}]^{+\cdot}\right.$ detected).

\subsubsection{2-(4-(Diphenylamino)Benzylidene)-1H-Cyclopenta[b]Naphthalene-1,3(2H)-Dione PP6}

4-(Diphenylamino)benzaldehyde $(0.70 \mathrm{~g}, 2.55 \mathrm{mmol}), \mathrm{m}_{\exp }=1.02 \mathrm{~g}, 2.26 \mathrm{mmol}, 89 \%$ yield. ${ }^{1} \mathrm{H} \mathrm{nmR}$ $\left(\mathrm{CDCl}_{3}\right)$ 8: $7.02(\mathrm{~d}, 2 \mathrm{H}, \mathrm{J}=8.9 \mathrm{~Hz}), 7.20-7.24(\mathrm{~m}, 6 \mathrm{H}), 7.38(\mathrm{t}, 4 \mathrm{H}, \mathrm{J}=8.2 \mathrm{~Hz}), 7.65-7.67(\mathrm{~m}, 2 \mathrm{H}), 7.88$ (s, $1 H), 8.07-8.09(\mathrm{~m}, 2 \mathrm{H}), 8.44(\mathrm{~d}, 2 \mathrm{H}, \mathrm{J}=7.7 \mathrm{~Hz}), 8.50(\mathrm{~d}, 2 \mathrm{H}, \mathrm{J}=8.9 \mathrm{~Hz}) ;{ }^{13} \mathrm{C} \mathrm{nmR}\left(\mathrm{CDCl}_{3}\right) \delta: 118.7$, 123.50, 123.56, 125.7, 126.0, 126.7, 127.2, 128.8, 128.9, 129.8, 130.3, 130.4, 135.8, 136.3, 136.4, 137.3, 137.7, 145.6, 147.6, 153.1, 189.4, and 191.0; HRMS (ESI MS) m/z: theor: 451.1572 found: 451.1574 $\left([\mathrm{M}]^{+} \cdot\right.$ detected).

\subsubsection{2-(2-Butoxy-4-(Diethylamino)Benzylidene)- $1 H$-Cyclopenta[ $b]$ Naphthalene-1,3(2H)-Dione PP7}

4-(Bis(4-bromophenyl)amino)benzaldehyde (1.1 g, $2.55 \mathrm{mmol}, \mathrm{M}=431.13 \mathrm{~g} / \mathrm{mol}), \mathrm{m}_{\exp }=1.42 \mathrm{~g}$, $2.33 \mathrm{mmol}, 92 \%$ yield. ${ }^{1} \mathrm{H} \mathrm{nmR}\left(\mathrm{CDCl}_{3}\right) \delta: 7.03-7.08(\mathrm{~m}, 6 \mathrm{H}), 7.48(\mathrm{~d}, 4 \mathrm{H}, \mathrm{J}=8.7 \mathrm{~Hz}), 7.66-7.69(\mathrm{~m}, 2 \mathrm{H})$, $7.88(\mathrm{~s}, 1 H), 8.07-8.11(\mathrm{~m}, 2 H), 8.46-8.52(\mathrm{~m}, 4 H) ;{ }^{13} \mathrm{C} \mathrm{nmR}\left(\mathrm{CDCl}_{3}\right) \delta: 118.6,119.8,123.78,123.82,127.1$, 127.7, 128.2, 128.98, 129.08, 133.0, 135.7, 136.4, 136.5, 137.0, 137.6, 144.7, 147.0, 151.9, 189.3, and 190.7; HRMS (ESI MS) m/z: theor: 606.9783 found: 606.9787 ([M] $^{+\cdot}$ detected).

\subsubsection{2-((9-Methyl-9H-Carbazol-3-Yl)Methylene)-1H-Cyclopenta[b]Naphthalene-1,3(2H)-Dione PP8}

9-Methyl-9H-carbazole-3-carbaldehyde $(0.53 \mathrm{~g}, 2.55 \mathrm{mmol}), \mathrm{m}_{\exp }=0.84 \mathrm{~g}, 2.17 \mathrm{mmol}, 85 \%$ yield. ${ }^{1} \mathrm{H} \mathrm{nmR}\left(\mathrm{CDCl}_{3}\right) \delta: 3.91(\mathrm{~s}, 3 H), 7.37(\mathrm{t}, 1 \mathrm{H}, \mathrm{J}=6.7 \mathrm{~Hz}), 7.43(\mathrm{~d}, 1 \mathrm{H}, \mathrm{J}=7.8 \mathrm{~Hz}), 7.48(\mathrm{~d}, 1 \mathrm{H}, \mathrm{J}=8.7$ $\mathrm{Hz}), 7.53(\mathrm{t}, 1 \mathrm{H}, \mathrm{J}=7.3 \mathrm{~Hz}), 7.66-7.69(\mathrm{~m}, 2 H), 8.05-8.12(\mathrm{~m}, 2 H), 8.20(\mathrm{~s}, 1 H), 8.28(\mathrm{~d}, 1 \mathrm{H}, \mathrm{J}=7.3 \mathrm{~Hz})$, $8.48(\mathrm{~d}, 2 \mathrm{H}, \mathrm{J}=7.6 \mathrm{~Hz}), 8.76(\mathrm{~d}, 1 \mathrm{H}, \mathrm{J}=8.0 \mathrm{~Hz}) ;{ }^{13} \mathrm{C} \mathrm{nmR}\left(\mathrm{CDCl}_{3}\right) \delta: 29.4,108.8,109.2,120.8,121.0$, 123.2, 123.66, 123.68, 123.7, 125.3, 126.8, 127.5, 128.8, 128.9, 129.0, 130.38, 130.40, 133.9, 135.7, 136.3, 136.5, 137.8, 141.7, 144.5, 149.9, 189.5, and 190.9; HRMS (ESI MS) m/z: theor: 387.1259 found: 387.1263 $\left([\mathrm{M}]^{+} \cdot\right.$ detected).

\subsubsection{2-(3-(4-(Dimethylamino)Phenyl)Allylidene)- $1 H$-Cyclopenta[b]Naphthalene-1,3(2H)-Dione PP9}

3-(4-(Dimethylamino)phenyl)acrylaldehyde (0.45 g, $2.55 \mathrm{mmol}), \mathrm{m}_{\exp }=756 \mathrm{mg}, 2.14 \mathrm{mmol}, 84 \%$ yield. ${ }^{1} \mathrm{H} \mathrm{nmR}\left(\mathrm{CDCl}_{3}\right) \delta: 3.10(\mathrm{~s}, 6 \mathrm{H}), 6.70(\mathrm{~d}, 2 \mathrm{H}, \mathrm{J}=8.4 \mathrm{~Hz}), 7.36(\mathrm{~d}, 1 \mathrm{H}, \mathrm{J}=15.1 \mathrm{~Hz}), 7.61-7.75$ $(\mathrm{m}, 4 H), 7.73(\mathrm{~d}, 1 \mathrm{H}, \mathrm{J}=12.3 \mathrm{~Hz}), 8.03-8.07(\mathrm{~m}, 2 \mathrm{H}), 8.34-8.39(\mathrm{~m}, 3 H) ;{ }^{13} \mathrm{C} \mathrm{nmR}\left(\mathrm{CDCl}_{3}\right) \delta: 40.1,111.9$, 119.8, 122.9, 123.3, 123.8, 126.3, 128.66, 128.72, 130.30, 130.35, 131.7, 136.2, 136.3, 136.5, 137.6, 147.6, 152.8, 154.8, 190.6, and 190.9; HRMS (ESI MS) m/z: theor: 353.1416 found: 353.1414 ([M] ${ }^{+\cdot}$ detected). 
2.2.10. 2-(3,3-Bis(4-(Dimethylamino)Phenyl)Allylidene)-1H-Cyclopenta[b]Naphthalene-1,3(2H)Dione PP10

3,3-Bis(4-(dimethylamino)phenyl)acrylaldehyde (0.75 g, $2.55 \mathrm{mmol}), \mathrm{m}_{\exp }=1.06 \mathrm{~g}, 2.24 \mathrm{mmol}$, $88 \%$ yield. ${ }^{1} \mathrm{H} \mathrm{nmR}\left(\mathrm{CDCl}_{3}\right) \delta: 3.08(\mathrm{~s}, 6 \mathrm{H}), 3.09(\mathrm{~s}, 6 \mathrm{H}), 6.68(\mathrm{~d}, 2 \mathrm{H}, \mathrm{J}=9.0 \mathrm{~Hz}), 6.78(\mathrm{~d}, 2 \mathrm{H}, \mathrm{J}=8.7$ $\mathrm{Hz}), 7.24(\mathrm{~d}, 2 \mathrm{H}, \mathrm{J}=10.2 \mathrm{~Hz}), 7.50(\mathrm{~d}, 2 \mathrm{H}, \mathrm{J}=8.9 \mathrm{~Hz}), 7.60-7.63(\mathrm{~m}, 2 \mathrm{H}), 7.78(\mathrm{~d}, 1 \mathrm{H}, \mathrm{J}=12.9 \mathrm{~Hz})$, 8.01-8.06 (m, 2H), $8.33(\mathrm{~d}, 2 \mathrm{H}, \mathrm{J}=5.2 \mathrm{~Hz}), 8.42(\mathrm{~d}, 2 \mathrm{H}, \mathrm{J}=12.9 \mathrm{~Hz}) ;{ }^{13} \mathrm{C} \mathrm{nmR}\left(\mathrm{CDCl}_{3}\right) \delta: 40.1,40.2$, 111.4, 111.5, 119.4, 122.4, 122.7, 125.8, 125.9, 128.3, 128.4, 128.8, 130.2, 132.5, 133.7, 136.1, 136.3, 136.7, 137.8, 146.6, 151.9, 152.4, 167.0, 191.0, and 191.1; HRMS (ESI MS) m/z: theor: 472.2151 found: 472.2148 $\left([\mathrm{M}]^{+} \cdot\right.$ detected).

\subsection{General Procedure for the Synthesis of PP11-PP20}

Indane-1,3-dione (1H-indene-1,3(2H)-dione) EA1 (0.37 g, $2.55 \mathrm{mmol})$ and the appropriate substituted benzaldehyde ( $2.55 \mathrm{mmol}, 1$ eq.) were dissolved in absolute ethanol $(50 \mathrm{~mL})$ and a few drops of piperidine were added. The reaction mixture was refluxed and progress of the reaction was followed by TLC. After cooling, a precipitate formed in most of the case. This latter was filtered off, washed several times with ethanol and dried under vacuum. For several chromophores (PP11, PP12, PP13 or PP20), a purification by column chromatography on $\mathrm{SiO}_{2}$ was required.

\subsubsection{2-(4-(Dodecyloxy)Benzylidene)-1H-Indene-1,3(2H)-Dione PP11}

4-(Dodecyloxy)benzaldehyde $(0.74 \mathrm{~g}, 2.55 \mathrm{mmol}), \mathrm{m}_{\exp }=790 \mathrm{mg}, 1.89 \mathrm{mmol}, 74 \%$ yield. ${ }^{1} \mathrm{H} \mathrm{nmR}$ $\left(\mathrm{CDCl}_{3}\right) \delta: 0.88(\mathrm{t}, 3 \mathrm{H}, \mathrm{J}=6.4 \mathrm{~Hz}), 1.21-1.54(\mathrm{~m}, 18 \mathrm{H}), 1.83(\mathrm{qt}, 2 \mathrm{H}, \mathrm{J}=7.8 \mathrm{~Hz}), 4.07(\mathrm{t}, 2 \mathrm{H}, \mathrm{J}=6.5 \mathrm{~Hz}), 7.01$ $(\mathrm{d}, 2 \mathrm{H}, \mathrm{J}=8.9 \mathrm{~Hz}), 7.77-7.85(\mathrm{~m}, 2 \mathrm{H}), 7.85(\mathrm{~s}, 1 H), 7.97-8.00(\mathrm{~m}, 2 \mathrm{H}), 8.54(\mathrm{~d}, 2 \mathrm{H}, \mathrm{J}=8.9 \mathrm{~Hz}) ;{ }^{13} \mathrm{C} \mathrm{nmR}$ $\left(\mathrm{CDCl}_{3}\right) \delta: 14.1,22.7,26.0,29.1,29.3,29.54,29.58,29.62,29.64,31.9,68.5,114.9,123.0,126.3,126.4,134.8$, 135.0, 137.3, 140.0, 142.4, 147.0, 163.8, 189.6, and 190.9; HRMS (ESI MS) m/z: theor: 418.2508 found: $418.2503\left([\mathrm{M}]^{+} \cdot\right.$ detected $)$.

\subsubsection{2-(3,4-Dibutoxybenzylidene)-1H-Indene-1,3(2H)-Dione PP12}

3,4-Dibutoxybenzaldehyde $(0.64 \mathrm{~g}, 2.55 \mathrm{mmol}), \mathrm{m}_{\exp }=820 \mathrm{mg}, 2.17 \mathrm{mmol}, 85 \%$ yield. ${ }^{1} \mathrm{H} \mathrm{nmR}$ $\left(\mathrm{CDCl}_{3}\right) \delta: 1.00(\mathrm{t}, 3 \mathrm{H}, \mathrm{J}=7.5 \mathrm{~Hz}), 1.02(\mathrm{t}, 3 \mathrm{H}, \mathrm{J}=7.5 \mathrm{~Hz}), 1.48-1.63(\mathrm{~m}, 4 \mathrm{H}), 1.83-1.93(\mathrm{~m}, 4 \mathrm{H}), 4.12$ $(\mathrm{t}, 2 \mathrm{H}, \mathrm{J}=6.6 \mathrm{~Hz}), 4.23(\mathrm{t}, 2 \mathrm{H}, \mathrm{J}=6.4 \mathrm{~Hz}), 6.95(\mathrm{~d}, 1 \mathrm{H}, \mathrm{J}=8.5 \mathrm{~Hz}), 7.77-7.82(\mathrm{~m}, 4 H), 7.96-8.00(\mathrm{~m}, 2 \mathrm{H})$, $8.72(\mathrm{~d}, 1 \mathrm{H}, \mathrm{J}=1.7 \mathrm{~Hz}) ;{ }^{13} \mathrm{C} \mathrm{nmR}\left(\mathrm{CDCl}_{3}\right) \delta: 13.8,13.9,19.2,19.3,31.0,31.2,68.8,68.9,112.1,117.6,123.0$, 126.2, 126.6, 131.2, 134.7, 134.9, 140.0, 142.5, 147.6, 148.8, 154.4, 189.7, and 190.9; HRMS (ESI MS) m/z: theor: 378.1831 found: $378.1832\left([\mathrm{M}]^{+\cdot}\right.$ detected).

\subsubsection{2-(2,4-Dibutoxybenzylidene)-1H-Indene-1,3(2H)-Dione PP13}

2,4-Dibutoxybenzaldehyde $(0.64 \mathrm{~g}, 2.55 \mathrm{mmol}), \mathrm{m}_{\exp }=723 \mathrm{mg}, 1.91 \mathrm{mmol}, 75 \%$ yield. ${ }^{1} \mathrm{H} \mathrm{nmR}$ $\left(\mathrm{CDCl}_{3}\right) \delta: 1.00(\mathrm{t}, 3 \mathrm{H}, \mathrm{J}=7.4 \mathrm{~Hz}), 1.02(\mathrm{t}, 3 \mathrm{H}, \mathrm{J}=7.3 \mathrm{~Hz}), 1.46-1.64(\mathrm{~m}, 6 \mathrm{H}), 1.76-1.94(\mathrm{~m}, 4 \mathrm{H}), 4.07$ $(\mathrm{t}, 2 \mathrm{H}, \mathrm{J}=6.4 \mathrm{~Hz}), 4.08(\mathrm{t}, 2 \mathrm{H}, \mathrm{J}=6.4 \mathrm{~Hz}), 6.42(\mathrm{~d}, 1 \mathrm{H}, \mathrm{J}=2.2 \mathrm{~Hz}), 6.61(\mathrm{dd}, 1 \mathrm{H}, \mathrm{J}=9.0 \mathrm{~Hz}, \mathrm{~J}=2.2 \mathrm{~Hz})$, 7.73-7.76 (m, 2H), 7.94-7.97 (m, 2H), $8.51(\mathrm{~s}, 1 H), 9.22(\mathrm{~d}, 1 \mathrm{H}, \mathrm{J}=9.0 \mathrm{~Hz}) ;{ }^{13} \mathrm{C} \mathrm{nmR}\left(\mathrm{CDCl}_{3}\right) \delta: 13.7,13.8$, 19.2, 31.1, 31.2, 68.2, 68.6, 98.8, 106.1, 116.1, 122.7, 122.8, 125.2, 134.5, 124.6, 136.5, 140.0, 141.3, 142.3162.7, 166.1, 189.8, and 191.2; HRMS (ESI MS) m/z: theor: 378.1831 found: 378.1834 ([M] $]^{+\cdot}$ detected)

\subsubsection{2-(4-(Dimethylamino)Benzylidene)-1H-Indene-1,3(2H)-Dione PP14}

4-Dimethylaminobenzaldehyde ( $0.38 \mathrm{~g}, 2.55 \mathrm{mmol}), \mathrm{m}_{\exp }=580 \mathrm{mg}, 2.09 \mathrm{mmol}, 82 \%$ yield $)$. ${ }^{1} \mathrm{H} \mathrm{nmR}\left(\mathrm{CDCl}_{3}\right)$ 8: $3.14(\mathrm{~s}, 6 \mathrm{H}), 6.75(\mathrm{~d}, 2 \mathrm{H}, \mathrm{J}=9.2 \mathrm{~Hz}), 7.70-7.75(\mathrm{~m}, 2 \mathrm{H}), 7.78(\mathrm{~s}, 1 \mathrm{H}), 7.90-7.94$ $(\mathrm{m}, 2 \mathrm{H}), 8.54(\mathrm{~d}, 2 \mathrm{H}, \mathrm{J}=9.2 \mathrm{~Hz}) ;{ }^{13} \mathrm{C} \mathrm{nmR}\left(\mathrm{CDCl}_{3}\right) \delta: 40.1,111.4,122.1,122.48,122.49,123.1,134.1,134.3$, 137.9, 139.9, 142.3, 147.5, 154.0, 190.0, and 191.7; HRMS (ESI MS) m/z: theor: 277.1103 found: 277.1105 $\left([\mathrm{M}]^{+} \cdot\right.$ detected). 


\subsubsection{2-(2-Butoxy-4-(Diethylamino)Benzylidene)-1H-Indene-1,3(2H)-Dione PP15}

2-Butoxy-4-(diethylamino)-benzaldehyde ( $0.64 \mathrm{~g}, 2.55 \mathrm{mmol}$ ), $\mathrm{m}_{\exp }=886 \mathrm{mg}, 2.35 \mathrm{mmol}, 92 \%$ yield. ${ }^{1} \mathrm{H} \mathrm{nmR}\left(\mathrm{CDCl}_{3}\right) \delta: 1.03(\mathrm{t}, 3 \mathrm{H}, \mathrm{J}=7.4 \mathrm{~Hz}), 1.26(\mathrm{t}, 6 \mathrm{H}, \mathrm{J}=7.1 \mathrm{~Hz}), 1.60(\mathrm{qt}, 2 \mathrm{H}, \mathrm{J}=7.4 \mathrm{~Hz}), 1.90$ $(\mathrm{qt}, 2 \mathrm{H}, \mathrm{J}=8.0 \mathrm{~Hz}), 3.48(\mathrm{q}, 4 \mathrm{H}, \mathrm{J}=7.1 \mathrm{~Hz}), 4.06(\mathrm{t}, 2 \mathrm{H}, \mathrm{J}=6.4 \mathrm{~Hz}), 6.03(\mathrm{~d}, 1 \mathrm{H}, \mathrm{J}=1.7 \mathrm{~Hz}), 6.40(\mathrm{dd}$, $1 H, \mathrm{~J}=9.3 \mathrm{~Hz}, \mathrm{~J}=1.7 \mathrm{~Hz}), 7.66-7.70(\mathrm{~m}, 2 \mathrm{H}), 7.85-7.89(\mathrm{~m}, 2 \mathrm{H}), 8.48(\mathrm{~s}, 1 \mathrm{H}), 9.35(\mathrm{~d}, 1 \mathrm{H}, \mathrm{J}=9.3 \mathrm{~Hz})$; ${ }^{13} \mathrm{C} \mathrm{nmR}\left(\mathrm{CDCl}_{3}\right)$ ): 12.8, 13.9, 19.4, 31.1, 45.0, 68.2, 93.2, 104.9, 112.5, 121.1, 122.1, 122.2, 133.6, 133.9, 137.3, 139.9, 141.1, 142.2, 154.7, 163.7, 190.3, and 192.2; HRMS (ESI MS) m/z: theor: 377.1991 found: $377.1992\left([\mathrm{M}]^{+} \cdot\right.$ detected).

\subsubsection{2-(4-(Diphenylamino)Benzylidene)-1H-Indene-1,3(2H)-Dione PP16}

4-(Diphenylamino)benzaldehyde $(0.70 \mathrm{~g}, 2.55 \mathrm{mmol}) \mathrm{m}_{\exp }=890 \mathrm{mg}, 2.22 \mathrm{mmol}, 87 \%$ yield. ${ }^{1} \mathrm{H} \mathrm{nmR}\left(\mathrm{CDCl}_{3}\right) \delta: 7.01(\mathrm{~d}, 2 \mathrm{H}, \mathrm{J}=8.9 \mathrm{~Hz}), 7.18-7.22(\mathrm{~m}, 6 \mathrm{H}), 7.34-7.38(\mathrm{~m}, 4 \mathrm{H}), 7.74-7.76(\mathrm{~m}, 2 \mathrm{H}), 7.78$ $(\mathrm{s}, 1 H), 7.94-7.96(\mathrm{~m}, 2 \mathrm{H}), 8.41(\mathrm{~d}, 2 \mathrm{H}, \mathrm{J}=8.9 \mathrm{~Hz}) ;{ }^{13} \mathrm{C} \mathrm{nmR}\left(\mathrm{CDCl}_{3}\right) \delta: 118.9,122.81,122.84,125.4,125.5$, $125.8,126.6,129.7,134.5,134.7,136.8,140.0,142.4,145.8,146.5,152.7,189.6$, and 191.2; HRMS (ESI MS) m/z: theor: 401.1416 found: $401.1418\left([\mathrm{M}]^{+}\right.$detected).

\subsubsection{2-(4-(Bis(4-Bromophenyl)Amino)Benzylidene)-1H-Indene-1,3(2H)-Dione PP17}

4-(bis(4-bromophenyl)amino)benzaldehyde (1.1 g, $2.55 \mathrm{mmol}), \mathrm{m}_{\exp }=1.15 \mathrm{~g}, 2.06 \mathrm{mmol}, 81 \%$ yield. ${ }^{1} \mathrm{H} \mathrm{nmR}\left(\mathrm{CDCl}_{3}\right)$ 8: 7.02-7.07 $(\mathrm{m}, 6 \mathrm{H}), 7.46(\mathrm{~d}, 4 \mathrm{H}, \mathrm{J}=8.8 \mathrm{~Hz}), 7.76-7.78(\mathrm{~m}, 3 \mathrm{H}), 7.96-7.98(\mathrm{~m}$, $2 \mathrm{H}), 8.42(\mathrm{~d}, 2 \mathrm{H}, \mathrm{J}=8.9 \mathrm{~Hz}) ;{ }^{13} \mathrm{C} \mathrm{nmR}\left(\mathrm{CDCl}_{3}\right) \delta: 118.4,120.0,122.98,123.01,126.4,126.9,127.6,133.0$, 134.7, 135.0, 136.6, 140.1, 142.4, 144.8, 146.0, 151.5, 189.5, and 190.9; HRMS (ESI MS) m/z: theor: 556.9626 found: $556.9628\left([\mathrm{M}]^{+} \cdot\right.$ detected $)$.

\subsubsection{2-((9-Methyl-9H-Carbazol-3-Yl)Methylene)-1H-Indene-1,3(2H)-Dione PP18}

9-Methyl-9H-carbazole-3-carbaldehyde ( $0.54 \mathrm{~g}, 2.55 \mathrm{mmol}), \mathrm{m}_{\exp }=671 \mathrm{mg}, 1.99 \mathrm{mmol}, 78 \%$ yield; ${ }^{1} \mathrm{H} \mathrm{nmR}\left(\mathrm{CDCl}_{3}\right)$ 8: $3.91(\mathrm{~s}, 3 \mathrm{H}), 7.35(\mathrm{t}, 1 \mathrm{H}, \mathrm{J}=7.5 \mathrm{~Hz}), 7.43-7.48(\mathrm{~m}, 2 \mathrm{H}), 7.54(\mathrm{t}, 1 \mathrm{H}, \mathrm{J}=7.3 \mathrm{~Hz})$, $7.76-7.81(\mathrm{~m}, 2 \mathrm{H}), 7.97-8.03(\mathrm{~m}, 2 \mathrm{H}), 8.25(\mathrm{~d}, 2 \mathrm{H}, \mathrm{J}=7.7 \mathrm{~Hz}), 8.69(\mathrm{~d}, 1 \mathrm{H}, \mathrm{J}=8.7 \mathrm{~Hz}) ;{ }^{13} \mathrm{C} \mathrm{nmR}\left(\mathrm{CDCl}_{3}\right)$ 8: 29.4, 108.7, 109.1, 120.7, 121.0, 122.9, 123.2, 123.6, 125.0, 125.6, 126.8, 128.6, 133.4, 134.6, 134.8, 140.0, 141.7, 142.5, 144.2, 148.8, 189.8, and 191.2; HRMS (ESI MS) m/z: theor: 337.1103 found: 337.1101 $\left([\mathrm{M}]^{+\cdot}\right.$ detected).

\subsubsection{2-(3-(4-(Dimethylamino)Phenyl)Allylidene)-1H-Indene-1,3(2H)-Dione PP19}

3-(4-(Dimethylamino)-phenyl)acrylaldehyde (0.45 g, $2.55 \mathrm{mmol}), \mathrm{m}_{\exp }=688 \mathrm{mg}, 2.27 \mathrm{mmol}, 89 \%$ yield. ${ }^{1} \mathrm{H} \mathrm{nmR}\left(\mathrm{CDCl}_{3}\right)$ 8: $3.09(\mathrm{~s}, 6 \mathrm{H}), 6.69(\mathrm{~d}, 2 \mathrm{H}, \mathrm{J}=8.9 \mathrm{~Hz}), 7.31(\mathrm{~d}, 1 \mathrm{H}, \mathrm{J}=15.1 \mathrm{~Hz}), 7.59(\mathrm{~d}, 2 \mathrm{H}, \mathrm{J}=8.4$ $\mathrm{Hz}), 7.64(\mathrm{~d}, 1 \mathrm{H}, \mathrm{J}=12.3 \mathrm{~Hz}), 7.71-7.74(\mathrm{~m}, 2 H), 7.90-7.92(\mathrm{~m}, 2 H), 8.28(\mathrm{dd}, 1 \mathrm{H}, \mathrm{J}=15.1 \mathrm{~Hz}, \mathrm{~J}=12.3 \mathrm{~Hz})$; ${ }^{13} \mathrm{C} \mathrm{nmR}\left(\mathrm{CDCl}_{3}\right)$ 8: 40.1, 111.9, 119.4, 122.4, 122.6, 123.8, 124.5, 131.3, 134.3, 134.4, 140.8, 142.1, 146.5, 152.5, 153.6, 190.8, and 191.2; HRMS (ESI MS) m/z: theor: 303.1259 found: 303.1255 ([M] ${ }^{+\cdot}$ detected).

\subsubsection{2-(3,3-Bis(4-(Dimethylamino)Phenyl)Allylidene)-1H-Indene-1,3(2H)-Dione PP20}

3-(4-(Dimethylamino)phenyl)-acrylaldehyde ( $0.75 \mathrm{~g}, 2.55 \mathrm{mmol}), \mathrm{m}_{\exp }=915 \mathrm{mg}, 2.16 \mathrm{mmol}, 85 \%$ yield. ${ }^{1} \mathrm{H} \mathrm{nmR}\left(\mathrm{CDCl}_{3}\right) \delta: 3.06(\mathrm{~s}, 6 \mathrm{H}), 3.07(\mathrm{~s}, 6 \mathrm{H}), 6.67(\mathrm{~d}, 2 \mathrm{H}, \mathrm{J}=9.0 \mathrm{~Hz}), 6.76(\mathrm{~d}, 2 \mathrm{H}, \mathrm{J}=8.8 \mathrm{~Hz}), 7.20$ $(\mathrm{d}, 2 \mathrm{H}, \mathrm{J}=8.7 \mathrm{~Hz}), 7.45(\mathrm{~d}, 2 \mathrm{H}, \mathrm{J}=9.0 \mathrm{~Hz}), 7.67-7.71(\mathrm{~m}, 3 \mathrm{H}), 7.84-7.89(\mathrm{~m}, 2 \mathrm{H}), 8.28(\mathrm{~d}, 1 \mathrm{H}, \mathrm{J}=12.8 \mathrm{~Hz})$; ${ }^{13} \mathrm{C} \mathrm{nmR}\left(\mathrm{CDCl}_{3}\right) \delta: 40.1,40.2,111.4,111.5,118.8,122.1,122.3,123.9,125.7,128.8,130.7,132.1,132.7$, 133.4, 133.9, 134.1, 140.7, 142.0, 145.5, 151.7, 152.1, 165.5, 191.3, and 191.5; HRMS (ESI MS) m/z: theor: 422.1994 found: $422.1998\left([\mathrm{M}]^{+} \cdot\right.$ detected). 


\section{Results and Discussion}

\subsection{Synthesis of the Dyes and Electron Acceptors}

All dyes PP1-PP20 presented in this work have been synthesized by a Knoevenagel reaction involving the aldehydes D1-D10 and the two electron acceptors EA4 and EA1, respectively (See Figure 3). The different reactions were performed in ethanol using piperidine as the catalyst. PP1-PP20 were obtained with reaction yields ranging from 74\% yield for PP4 and PP11 to $94 \%$ for PP5 (see Table 1). All compounds were obtained as solids and they were characterized by ${ }^{1} \mathrm{H},{ }^{13} \mathrm{C} \mathrm{nmR}$ spectroscopies, and HRMS spectrometry (see Supplementary Materials). It has to be noticed that the synthetic procedure to EA4 has been greatly improved compared to that reported in the literature [47], enabling to reach a reaction yield of $91 \%$. Indeed, a common method to synthesize 1,3-indanedione derivatives consists in a Claisen condensation of the corresponding diesters with ethyl acetate under basic conditions (generally sodium hydride).
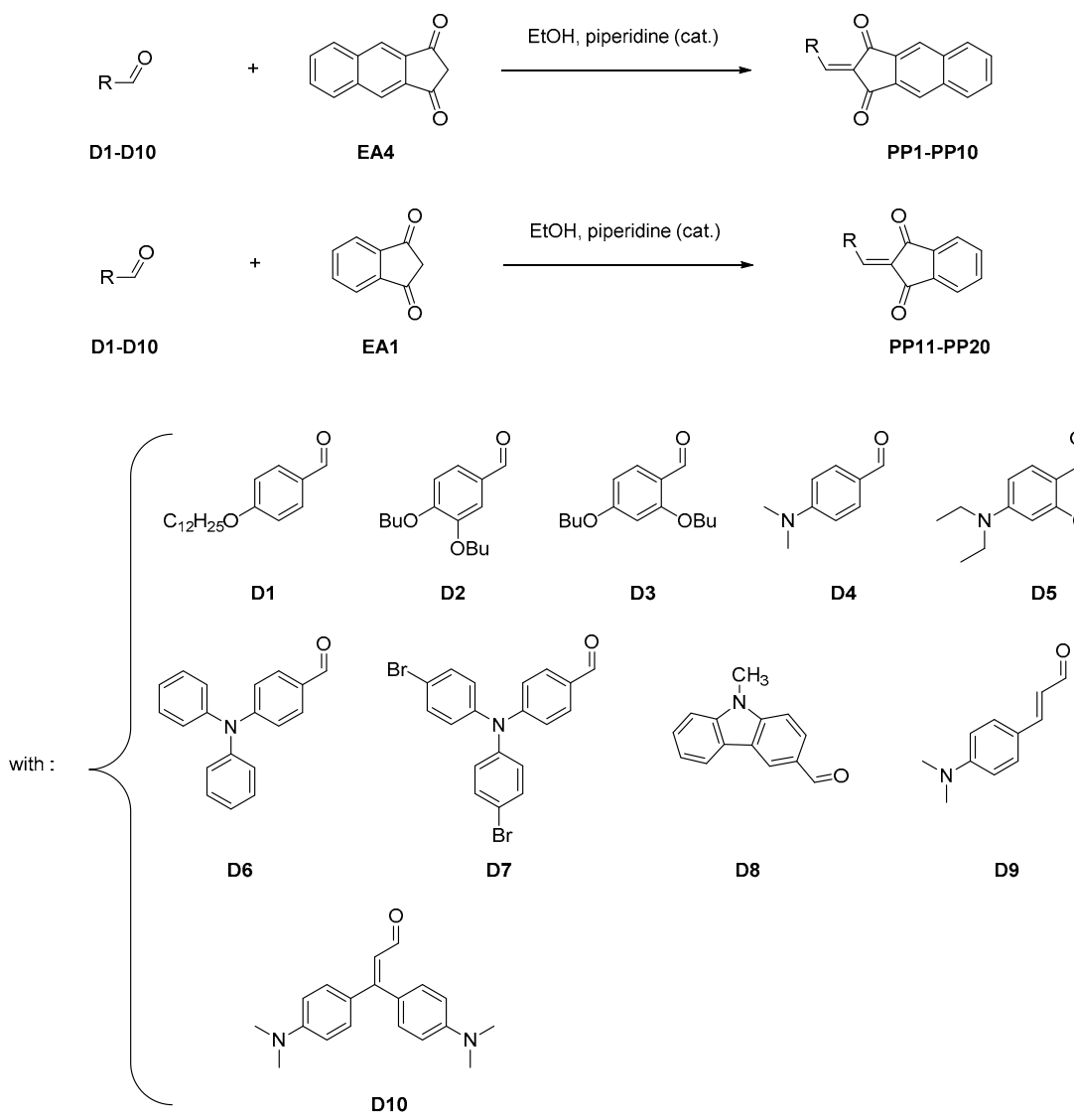

Figure 3. Synthetic pathways to PP1-PP20 and the ten aldehydes used in this study.

Table 1. Reaction yields obtained for the synthesis of PP1-PP20.

\begin{tabular}{ccccccccccc}
\hline Compounds & PP1 & PP2 & PP3 & PP4 & PP5 & PP6 & PP7 & PP8 & PP9 & PP10 \\
\hline Reaction yields (\%) & 88 & 84 & 88 & 74 & 94 & 89 & 92 & 85 & 84 & 88 \\
\hline Compounds & PP11 & PP12 & PP13 & PP14 & PP15 & PP16 & PP17 & PP18 & PP19 & PP20 \\
\hline Reaction yields (\%) & 74 & 85 & 75 & 82 & 92 & 87 & 81 & 78 & 89 & 85 \\
\hline
\end{tabular}

The final product is obtained after the condensation step by decarboxylation of the intermediate salt under hot acidic conditions (see Figure 4). To access the starting compound, i.e., diethyl naphthalene-2,3-dicarboxylate, two different reaction pathways were examined, by esterification of 
naphthalene-2,3-dicarboxylic acid in ethanol in the presence of an excess of thionyl chloride, or by the classical esterification conditions consisting in refluxing the acid in ethanol in the presence of a catalytic amount of $\mathrm{H}_{2} \mathrm{SO}_{4}$ (see Figure 4). If diethyl naphthalene-2,3-dicarboxylate could be obtained in almost quantitative yields with the two procedures, our attempt to convert the diester obtained by the first procedure were unfruitful to form EA4. This is attributable to remaining traces of $\mathrm{SOCl}_{2}$ in the diester, despites the numerous washings in basic conditions. Due to the presence of water traces in ethyl acetate, $\mathrm{SOCl}_{2}$ could react with water to form $\mathrm{HCl}$, neutralizing part of the $\mathrm{NaH}$ introduced. Conversely, in the second procedure, $\mathrm{H}_{2} \mathrm{SO}_{4}$ can be easily removed from the diester due to its catalytic use, avoiding this drawback. Using the oil obtained by this second procedure, a major improvement was obtained by replacing $\mathrm{NaH} 60 \%$ dispersion in oil by $\mathrm{NaH} 95 \%$ dispersion in oil for the first reaction step.

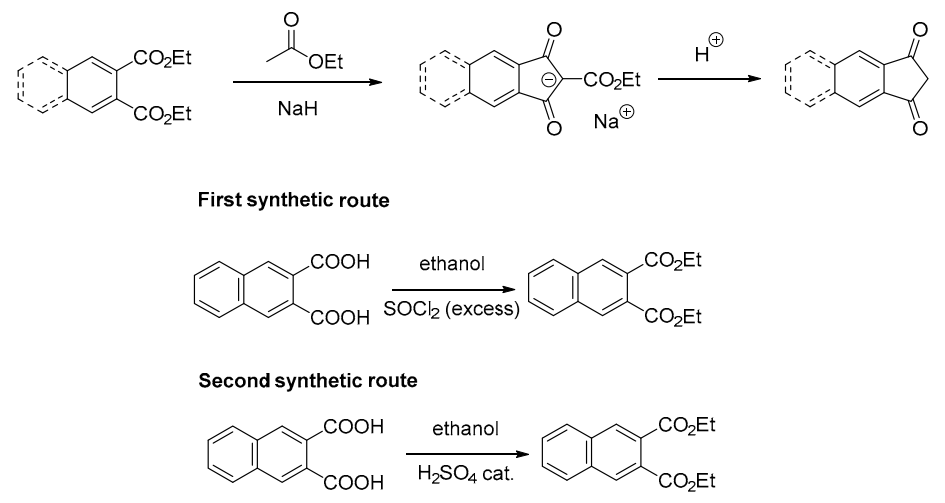

Figure 4. Synthetic route to 1,3-indanedione derivatives by the Claisen condensation and the two possible esterification procedures for naphthalene-2,3-dicarboxylic acid.

By using this more concentrated dispersion, the reaction yield of the crude materials for the two steps (the intermediate salt was not isolated) could be greatly increased. By modifying the decarboxylation time (increased from $1.5 \mathrm{~h}$ instead of $20 \mathrm{~min}$ ), the quantity of $\mathrm{NaH}$ ( 2.5 eq. instead of 1.45 eq.) and the recrystallization solvent [47] (benzene replaced by the less toxic toluene) compared to that reported in the literature, the overall reaction yield after purification could be increased from $65 \%$ (literature) up to $91 \%$ in our optimized conditions.

\subsection{Theoretical Calculations}

Theoretical studies were realized in order to investigate the energy levels as well as the molecular orbitals (M.O.) compositions of the different dyes. DFT calculations of all synthetized compounds were performed by the B3LYP/6-311G $(\mathrm{d}, \mathrm{p})$ level of theory using Gaussian 09 programs. Dichloromethane as the solvent and the polarizable continuum model (PCM) as the solvent model were used for the TD-DFT calculations. The optimized geometries as well as the highest occupied molecular orbitals (HOMO) and the lowest unoccupied molecular orbital (LUMO), i.e., the frontier orbitals' electronic distributions of selected compounds (PP6 and PP16) are given in Figure 5. The data for all compounds are supplied in the Supplementary Materials. The simulated absorption spectra are shown in the Figure 6 while the Table 2 summarizes all HOMO and LUMO energy levels and electronic transitions associated with the intramolecular charge transfer (ITC) absorption peaks. The assignment of the electronic transitions for $\lambda_{\max }$ reported in the Table 2 has been determined with GaussSum 3.0 software, and especially, the contribution of the different transitions. 


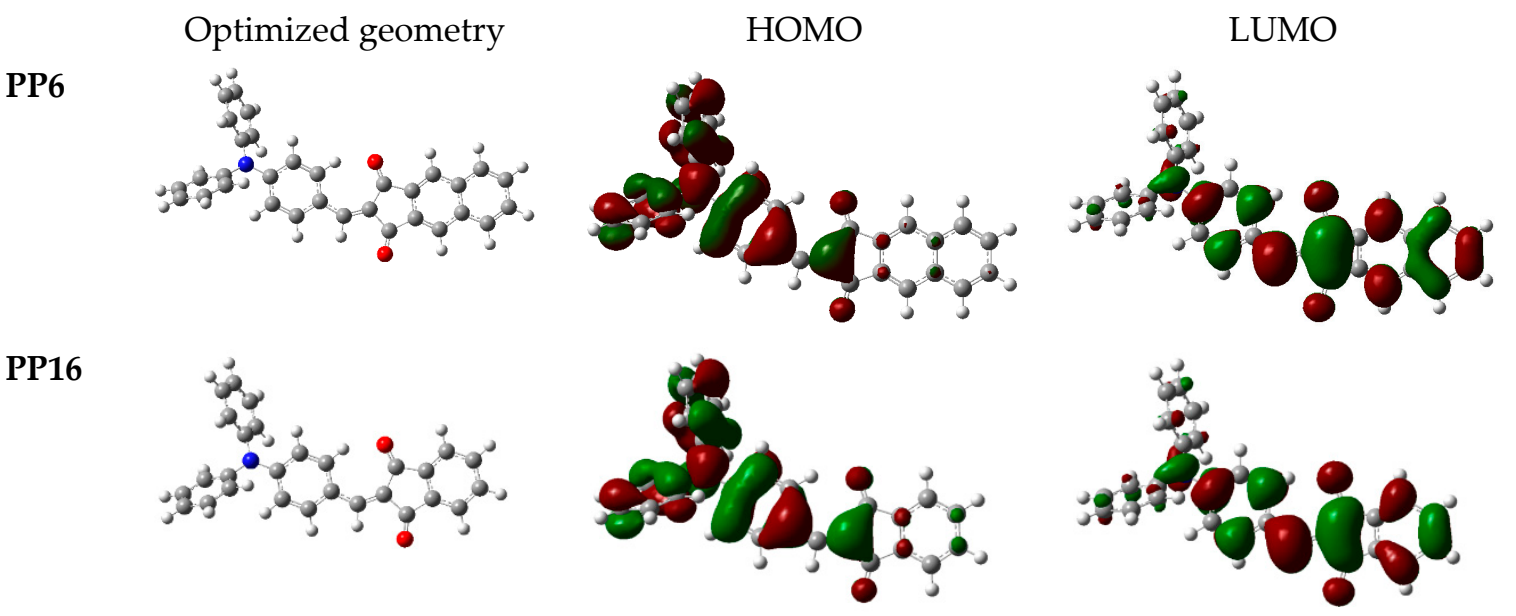

Figure 5. Optimized geometries and HOMO/LUMO electronic distributions of PP6 and PP16.

As can be observed for all compounds, clear common trends are well observed for the two series:

- ICT Absorption:

The absorption peaks corresponded to the ICT are well observed in the Figure 6 and the values of maximal ICT absorption wavelengths are given in the Table 2. A clear trend is observed in both series: The ICT transition is mainly associated with the HOMO-LUMO transition. When the electron donor properties of the donor part increase, a clear redshift is obtained for the ICT transition. When we compare the two molecules in the two series with the same donor part, we observe that the ICT of the EA4-based molecule is redshift compared to that of EA1-based counterpart (for instance: $\lambda_{\max }(\mathrm{nm})=518$ and 490 for PP6 and PP16, respectively, both compounds have triphenylamine as donor part).

- HOMO and LUMO Energy Levels:

Within the same series, the values of $\mathrm{E}_{\mathrm{HOMO}}$ importantly vary as a function of the electron donating capacity of the donor part while the values of $E_{L U M O}$ slightly change. When we compare cross the two series: two molecules with the same donor part, we observe that they have nearly the same $\mathrm{E}_{\mathrm{HOMO}}$ while the $\mathrm{E}_{\mathrm{LUMO}}$ of the EA4-based molecule is deeper compared to that of EA1-based counterpart, which is in good accordance with the more electron deficient nature of EA4 (for instance: $\mathrm{E}_{\mathrm{HOMO}}(\mathrm{eV})=-5.633$ and -5.635 for PP6 and PP16, respectively, while $\mathrm{E}_{\mathrm{LUMO}}(\mathrm{eV})=-2.836$ and -2.694 , respectively. Both compounds have triphenylamine as donor part).

- HOMO and LUMO Orbitals Distribution:

The HOMO orbitals are well developed over the electron donor part while the LUMO orbitals are on the electron deficient moiety. Only a small overlap between the HOMO and LUMO orbitals is detected (see Figure 5). 


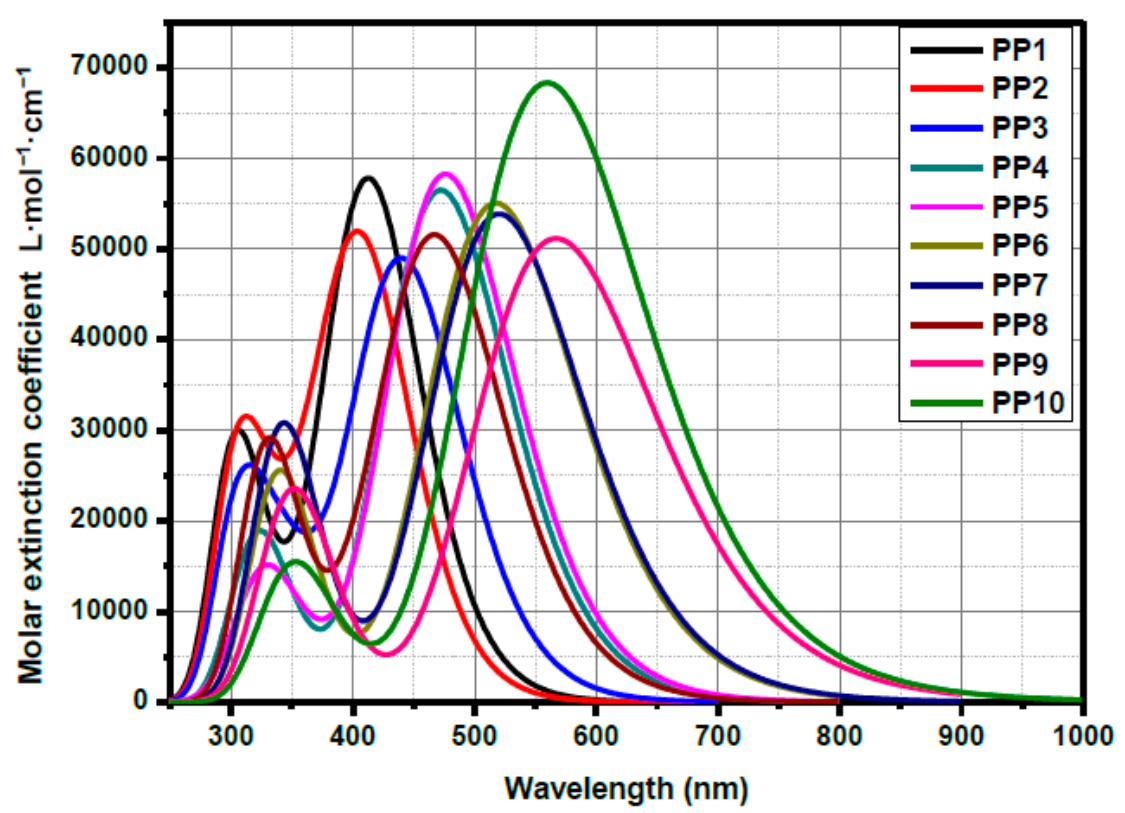

(a)

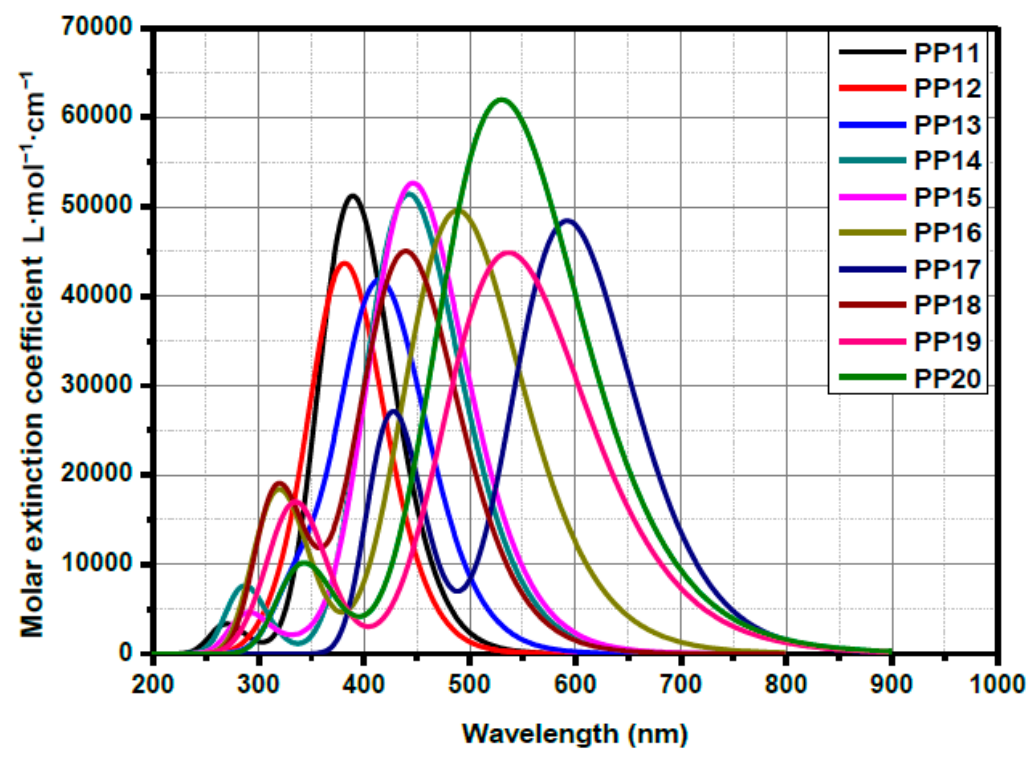

(b)

Figure 6. Simulated absorption spectra in dilute dichloromethane $\left(5 \times 10^{-3} \mathrm{M}\right)$ of synthetized compounds PP1-PP10 (a) and PP11-PP20 (b).

Table 2. Summary of simulated absorption characteristics in dilute dichloromethane of synthetized compounds. Data were obtained in dichloromethane solution.

\begin{tabular}{ccccc}
\hline Compounds & E $_{\text {HOMO }}(\mathbf{e V})$ & $\mathrm{E}_{\text {LUMO }}(\mathbf{e V})$ & $\lambda_{\max }(\mathbf{n m})$ & Transitions \\
\hline PP1 & -6.279 & -2.865 & 414 & HOMO->LUMO (95\%) \\
& & & & HOMO->LUMO (72\%) \\
PP2 & -6.468 & -2.983 & 406 & $\begin{array}{c}\text { HOMO-1->LUMO (22\%) } \\
\text { HOMO-2->LUMO (5\%) }\end{array}$ \\
PP3 & -5.974 & -2.691 & 441 & HOMO->LUMO (98\%) \\
PP4 & -5.717 & -2.668 & 473 & HOMO->LUMO (99\%) \\
\hline
\end{tabular}


Table 2. Cont.

\begin{tabular}{|c|c|c|c|c|}
\hline Compounds & $\mathrm{E}_{\text {HOMо }}(\mathrm{eV})$ & $E_{\text {LUMO }}(\mathrm{eV})$ & $\lambda_{\max }(\mathrm{nm})$ & Transitions \\
\hline PP5 & -5.522 & -2.489 & 477 & HOMO->LUMO (99\%) \\
\hline PP6 & -5.633 & -2.836 & 518 & HOMO->LUMO (99\%) \\
\hline PP7 & -5.826 & -3.003 & 520 & HOMO->LUMO (99\%) \\
\hline PP8 & -5.953 & -2.816 & 471 & HOMO->LUMO (99\%) \\
\hline PP9 & -5.554 & -2.874 & 567 & HOMO->LUMO (100\%) \\
\hline PP10 & -5.262 & -2.62 & 574 & $\begin{array}{c}\text { HOMO->LUMO }(90 \%) \\
\text { HOMO-1->LUMO }(10 \%)\end{array}$ \\
\hline PP11 & -6.3 & -2.71 & 390 & HOMO->LUMO (97\%) \\
\hline PP12 & -6.498 & -2.842 & 385 & $\begin{array}{l}\text { HOMO->LUMO }(94 \%) \\
\text { HOMO-1->LUMO }(4 \%)\end{array}$ \\
\hline PP13 & -5.982 & -2.526 & 417 & HOMO->LUMO (97\%) \\
\hline PP14 & -5.715 & -2.498 & 444 & $\begin{array}{l}\text { HOMO->LUMO }(95 \%) \\
\text { HOMO->L+1 }(4 \%)\end{array}$ \\
\hline PP15 & -5.513 & -2.309 & 448 & $\begin{array}{c}\text { HOMO->LUMO }(95 \%) \\
\text { HOMO->LUMO+1 }(4 \%)\end{array}$ \\
\hline PP16 & -5.635 & -2.694 & 490 & HOMO->LUMO (99\%) \\
\hline PP17 & -5.835 & -2.878 & 493 & HOMO->LUMO (99\%) \\
\hline PP18 & -5.962 & -2.656 & 443 & HOMO->LUMO (98\%) \\
\hline PP19 & -5.555 & -2.748 & 537 & HOMO->LUMO (100\%) \\
\hline PP20 & -5.253 & -2.489 & 545 & $\begin{array}{c}\text { HOMO->LUMO }(85 \%) \\
\text { HOMO-1->LUMO }(15 \%)\end{array}$ \\
\hline
\end{tabular}

\subsection{Optical Properties}

All compounds were characterized by UV-visible spectroscopy and their absorption spectra in dichloromethane are provided in the Figure 7. PP1-PP20 are push-pull compounds that possess an electron donating group and an electron accepting group which interact by mean of a $\pi$-conjugated system. The position of the charge transfer band will depend of parameters such as the strength of the electron donating/accepting groups, but also of the length of the $\pi$-conjugated system. By combining both effects, a decrease in the energy difference between the HOMO and the LUMO can be obtained, resulting in a shift of the absorption spectrum towards longer wavelengths.

While examining the first series PP1-PP10, absorption maxima ranging from $412 \mathrm{~nm}$ for PP1 to $524 \mathrm{~nm}$ for PP5 were found in dichloromethane for dyes exhibiting a short spacer. Elongation of the $\pi$-conjugated system resulted in a significant red-shift of the absorption maximum, shifting from $471 \mathrm{~nm}$ for PP1 to $571 \mathrm{~nm}$ for PP9. The most red-shifted absorption was found for PP10, peaking at $603 \mathrm{~nm}$. While comparing the PP1-PP10 series with PP11-PP20 based on 1,3-indanedione, absorption spectra of PP11-PP20 were found to follow the same order than that of the PP1-PP10 series, but with an absorption blue-shifted by about $30 \mathrm{~nm}$ (see Figure 7). Examination of the molar extinction coefficients for the two series also revealed the PP1-PP10 series to exhibit higher molar extinction coefficients than that of the PP11-PP20 series, consistent with an improvement of the molar absorptivity with the oscillator strength and the conjugation extension (see Figure 8) [48].

The experimental absorption spectra recorded in dichloromethane of all compounds are in good accordance with predicted properties obtained by DFT calculation. Notably, a good accordance between the theoretical absorption maxima can be found for all dyes (See Tables 2-4). Second, considering that the main absorption band was theoretically determined for all dyes originating from a HOMO->LUMO transition, this latter can thus be confidently assigned to the intramolecular charge transfer bands for all dyes. A contribution of the HOMO-> LUMO transition to the ICT band ranging between $85 \%$ (for PP20) to 100\% for PP9 and PP19 could be calculated. 


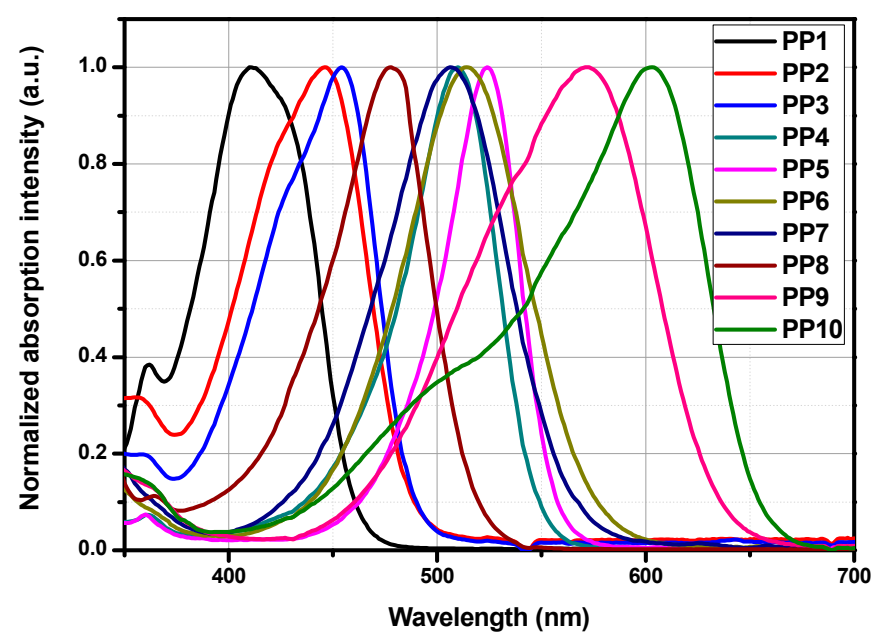

(a)

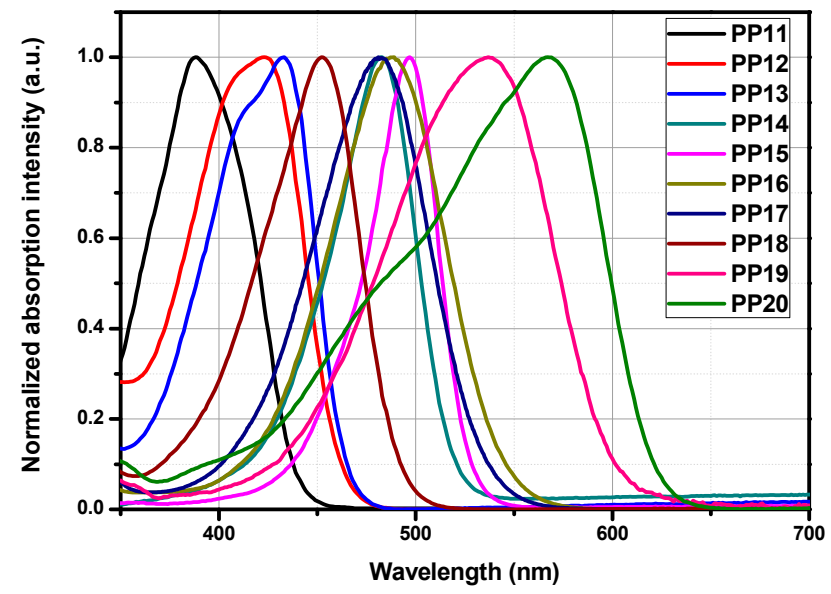

(b)

Figure 7. UV-visible absorption spectra of PP1-PP10 (a) and PP11-PP20 (b) in dichloromethane.

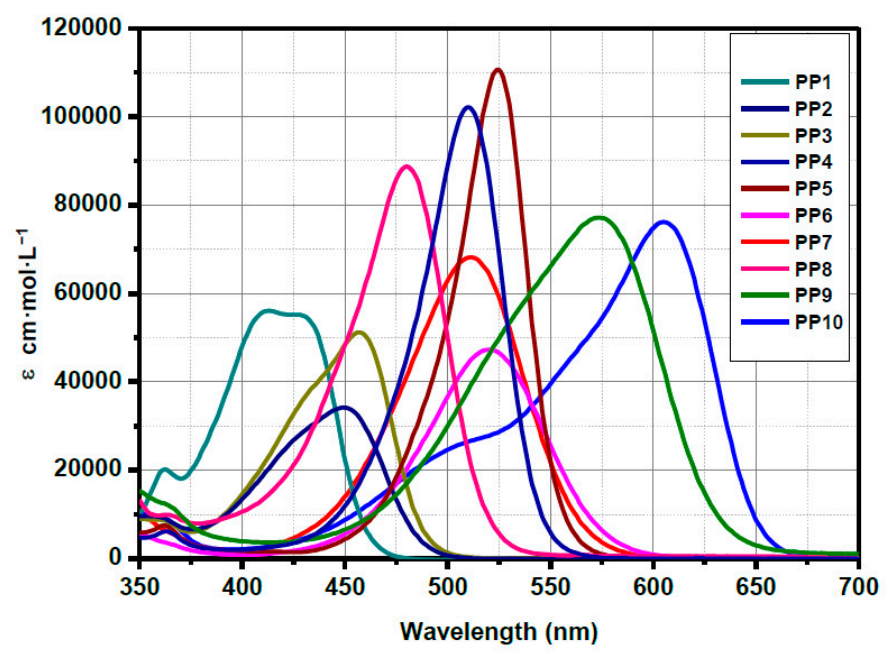

(a)

Figure 8. Cont. 


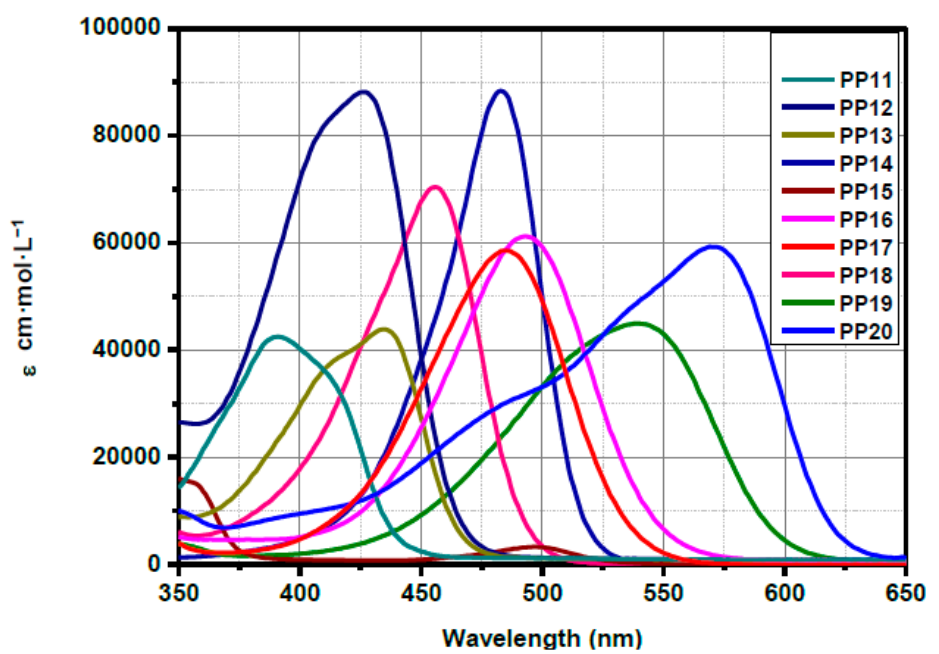

(b)

Figure 8. UV-visible absorption spectra of PP1-PP10 (a) and PP11-PP20 (b) in chloroform.

Table 3. Summary of the optical properties of PP1-PP10 in twenty-three solvents and Kamlet and Taft parameters $\pi^{*}$.

\begin{tabular}{cccccccccccc}
\hline Compounds & $\boldsymbol{\pi}^{* \mathbf{1}}$ & $\mathbf{P P 1}^{\mathbf{2}}$ & $\mathbf{P P 2}^{\mathbf{2}}$ & $\mathbf{P P 3}^{\mathbf{2}}$ & $\mathbf{P P 4}^{\mathbf{2}}$ & $\mathbf{P P 5}^{\mathbf{2}}$ & $\mathbf{P P 6}^{\mathbf{2}}$ & $\mathbf{P P 7}^{\mathbf{2}}$ & $\mathbf{P P 8}^{\mathbf{2}}$ & $\mathbf{P P 9}^{\mathbf{2}}$ & $\mathbf{P P 1 0}^{\mathbf{2}}$ \\
\hline diethyl ether & 0.27 & 403 & 442 & 445 & 490 & 505 & 499 & 489 & 464 & 534 & 569 \\
toluene & 0.54 & 405 & 450 & 441 & 501 & 512 & 509 & 501 & 473 & 553 & 582 \\
chloroform & 0.78 & 412 & 450 & 457 & 520 & 524 & 520 & 510 & 480 & 574 & 606 \\
THF & 0.58 & 405 & 444 & 450 & 502 & 517 & 504 & 497 & 471 & 555 & 587 \\
1,4-dioxane & 0.55 & 402 & 443 & 446 & 498 & 512 & 503 & 496 & 468 & 545 & 581 \\
acetone & 0.71 & 405 & 441 & 449 & 505 & 520 & 501 & 496 & 471 & 562 & 595 \\
dichloromethane & 0.82 & 411 & 448 & 456 & 510 & 524 & 514 & 505 & 476 & 571 & 603 \\
DMSO & 1.00 & 414 & 447 & 458 & 522 & 534 & 512 & 506 & 482 & 590 & 620 \\
heptane & -0.08 & 410 & 445 & 443 & 479 & 497 & 497 & 486 & 461 & 529 & 551 \\
acetonitrile & 0.75 & 404 & 439 & 450 & 507 & 523 & 505 & 496 & 471 & 565 & 597 \\
dimethylformamide & 0.87 & 409 & 447 & 454 & 514 & 528 & 509 & 503 & 478 & 579 & 609 \\
ethyl acetate & 0.54 & 404 & 444 & 447 & 498 & 513 & 500 & 491 & 468 & 546 & 579 \\
p-xylene & 0.43 & 406 & 448 & 448 & 496 & 509 & 504 & 497 & 470 & 547 & 580 \\
1,2-dichloroethane & 0.81 & 410 & 447 & 454 & 509 & 524 & 515 & 504 & 474 & 567 & 603 \\
dimethylacetamide & 0.88 & 410 & 447 & 456 & 516 & 529 & 508 & 502 & 477 & 577 & 608 \\
diglyme & 0.64 & 406 & 445 & 450 & 506 & 518 & 504 & 497 & 474 & 567 & 593 \\
cyclohexane & 0.00 & 412 & 446 & 445 & 482 & 499 & 502 & 492 & 462 & 532 & 556 \\
triethylamine & 0.14 & 439 & 454 & 450 & 486 & 501 & 503 & 507 & 463 & 533 & 559 \\
hexane & -0.08 & 409 & 444 & 442 & 477 & 495 & 497 & 488 & 457 & 525 & 551 \\
anisole & 0.73 & 413 & 450 & 454 & 508 & 520 & 514 & 504 & 476 & 567 & 599 \\
pentane & -0.09 & 408 & 442 & 440 & 476 & 495 & 495 & 485 & 458 & 524 & 548 \\
nitrobenzene & 1.01 & 433 & 452 & 461 & 518 & 532 & 519 & 509 & 484 & 585 & 613 \\
diethyl carbonate & 0.45 & 402 & 444 & 447 & 495 & 510 & 500 & 493 & 466 & 542 & 575 \\
\hline
\end{tabular}

${ }^{1}$ Kamlet and Taft parameters; ${ }^{2}$ Position of the ICT bands are given in $\mathrm{nm}$.

Table 4. Summary of the optical properties of PP11-PP20 in twenty-three solvents and Kamlet and Taft parameters $\pi^{*}$.

\begin{tabular}{|c|c|c|c|c|c|c|c|c|c|c|c|}
\hline Compounds & $\pi^{* 1}$ & PP11 $^{3}$ & $\mathrm{PP}^{2} 2^{3}$ & $\mathrm{PP}^{3} 3^{3}$ & PP14 3 & $\mathrm{PP}^{2} 5^{3}$ & PP16 ${ }^{3}$ & $\mathrm{PP}^{2} 7^{3}$ & PP18 3 & PP19 3 & $\mathrm{PP20}^{3}$ \\
\hline diethyl ether & 0.27 & 384 & 404 & 424 & 462 & 480 & 474 & 467 & 442 & 502 & 531 \\
\hline toluene & 0.54 & 388 & 408 & 429 & 474 & 486 & 485 & 476 & 451 & 520 & 552 \\
\hline chloroform & 0.78 & 391 & 416 & 436 & 483 & 497 & 493 & 485 & 456 & 539 & 571 \\
\hline $\mathrm{THF}$ & 0.58 & 387 & 411 & 428 & 476 & 490 & 479 & 472 & 448 & 518 & 553 \\
\hline
\end{tabular}


Table 4. Cont.

\begin{tabular}{|c|c|c|c|c|c|c|c|c|c|c|c|}
\hline Compounds & $\pi^{* 1}$ & PP11 $^{3}$ & $\mathrm{PP}^{2} 2^{3}$ & $\mathrm{PP}^{3} 3^{3}$ & $\mathrm{PP}_{14}^{3}$ & $\mathrm{PP}^{2} 5^{3}$ & $\mathrm{PP}^{3} 6^{3}$ & $\mathrm{PP}^{2} 7^{3}$ & PP18 3 & PP19 3 & $\mathrm{PP20}^{3}$ \\
\hline 1,4-dioxane & 0.55 & 385 & 406 & 426 & 471 & 486 & 474 & 471 & 444 & 512 & 549 \\
\hline acetone & 0.71 & 385 & 410 & 429 & 479 & 493 & 477 & 471 & 447 & 526 & 555 \\
\hline dichloromethane & 0.82 & 388 & 417 & 433 & 482 & 497 & 489 & 482 & 452 & 537 & 567 \\
\hline DMSO & 1.00 & 391 & 425 & 435 & 492 & 506 & 484 & 481 & 458 & 554 & 584 \\
\hline heptane & -0.08 & 379 & 400 & 422 & 457 & 473 & 476 & 468 & 439 & 478 & 523 \\
\hline acetonitrile & 0.75 & 383 & 410 & 428 & 480 & 494 & 477 & 471 & 446 & 528 & 556 \\
\hline DMF & 0.87 & 388 & 415 & 433 & 488 & 501 & 482 & 479 & 453 & 541 & 579 \\
\hline ethyl acetate & 0.54 & 385 & 409 & 426 & 474 & 486 & 474 & 470 & 447 & 520 & 544 \\
\hline p-xylene & 0.43 & 388 & 407 & 429 & 471 & 484 & 481 & 474 & 449 & 522 & 548 \\
\hline 1,2-dichloroethane & 0.81 & 388 & 421 & 432 & 481 & 496 & 487 & 480 & 453 & 536 & 567 \\
\hline dimethylacetamide & 0.88 & 388 & 412 & 432 & 489 & 500 & 485 & 478 & 454 & 543 & 573 \\
\hline diglyme & 0.64 & 386 & 412 & 429 & 479 & 490 & 480 & 472 & 450 & 524 & 559 \\
\hline cyclohexane & 0.00 & 382 & 402 & 423 & 459 & 474 & 478 & 471 & 441 & 481 & 527 \\
\hline triethylamine & 0.14 & 384 & 403 & 424 & 461 & 476 & 478 & 471 & 443 & 489 & 530 \\
\hline hexane & -0.08 & 378 & 400 & 422 & 455 & 471 & 474 & 467 & 437 & 476 & 521 \\
\hline anisole & 0.73 & 389 & 415 & 432 & 481 & 495 & 487 & 482 & 454 & 530 & 570 \\
\hline pentane & -0.09 & 378 & 399 & 420 & 454 & 470 & 474 & 465 & 436 & 474 & 520 \\
\hline nitrobenzene & 1.01 & n.d. ${ }^{2}$ & n.d. ${ }^{2}$ & 437 & 491 & 504 & 493 & 482 & 459 & 552 & 582 \\
\hline diethyl carbonate & 0.45 & 384 & 405 & 426 & 471 & 484 & 475 & 470 & 445 & 510 & 541 \\
\hline
\end{tabular}

${ }^{1}$ Kamlet and Taft parameters; ${ }^{2}$ not determined. ${ }^{3}$ Position of the ICT bands are given in $\mathrm{nm}$.

\subsection{Solvatochromism}

All dyes PP1-PP20 exhibited a good solubility in most of the common organic solvents so that examination of the solvatochromism could be carried out in 23 solvents of different polarities. It has to be noticed that alcohols such as methanol, ethanol, propan-2-ol, butan-1-ol and pentan-1-ol were initially considered as solvents for the solvatochromic study, but the absorption maxima obtained with these solvents were irregular compared to that obtained with the 23 other solvents. This specific behavior can be assigned to the fact that all dyes precipitated in alcohols such as in ethanol which was the solvent of reaction. Even if the absorption spectra could be recorded in alcohols for all dyes, presence of free molecules and aggregates in solution certainly modify the position of the absorption maxima. A summary of the absorption maxima for the twenty dyes are provided in Tables 3 and 4 .

As evidenced in the Tables 3 and 4, analysis of the solvatochromism in solvents of different polarities confirmed the presence of an intramolecular charge transfer in all dyes. Intramolecular nature of the charge transfer was demonstrated by performing successive dilutions, intensity of the charge transfer band linearly decreasing with the dye concentrations. Various empirical polarity scales have been developed over the years to interpret the solvent-solute interaction and the Kamlet-Taft's [49], Dimroth-Reichardt's [50], Lippert-Mataga's [51], Catalan's [52], Kawski-Chamma-Viallet's [53], McRae's [54], Suppan's [55], and Bakhshiev's [56] scales can be cited as the most popular ones. Among all scales, the Kamlet-Taft solvent polarity scale proved to be the most adapted one, linear correlations being obtained for all dyes by plotting the absorption maximum vs. The empirical Taft parameters (see linear regression in Supplementary Materials). For all the other polarity scales based on the dielectric constant or the refractive index of solvents, no reasonable correlations could be established. The Kamlet and Taft equation is also a multiparametric equation that can take into account the dipolarity-polarizability $\left(\pi^{*}\right)$, the hydrogen-donating and accepting ability ( $\alpha$ and $\beta$ ) of the solvents, modelizing more precisely the interactions between the solvent and the solute. However, multiple linear regression analyses carried out on the triparametric Kamlet-Taft equation using the three solvent descriptors $\left(\alpha, \beta, \pi^{*}\right)$ did not improve the correlation coefficients, as demonstrated in the Table in SI. It can therefore be concluded that the dipolarity-polarizability of the solvent is the primary cause influencing the position of the ICT band. 
As evidenced in the Figure 9 and Figures in Supplementary Materials, PP1-PP20 show negative slopes with good linear correlations, indicative of a positive solvatochromism. Excepted for PP1, PP2, PP11, and PP12 that possess weak electron donors, all dyes displayed strong negative slopes, indicative of a significant charge redistribution upon excitation. The most important solvatochromism was found for PP9, PP10, PP19, and PP20 that exhibit the longest conjugated spacers.

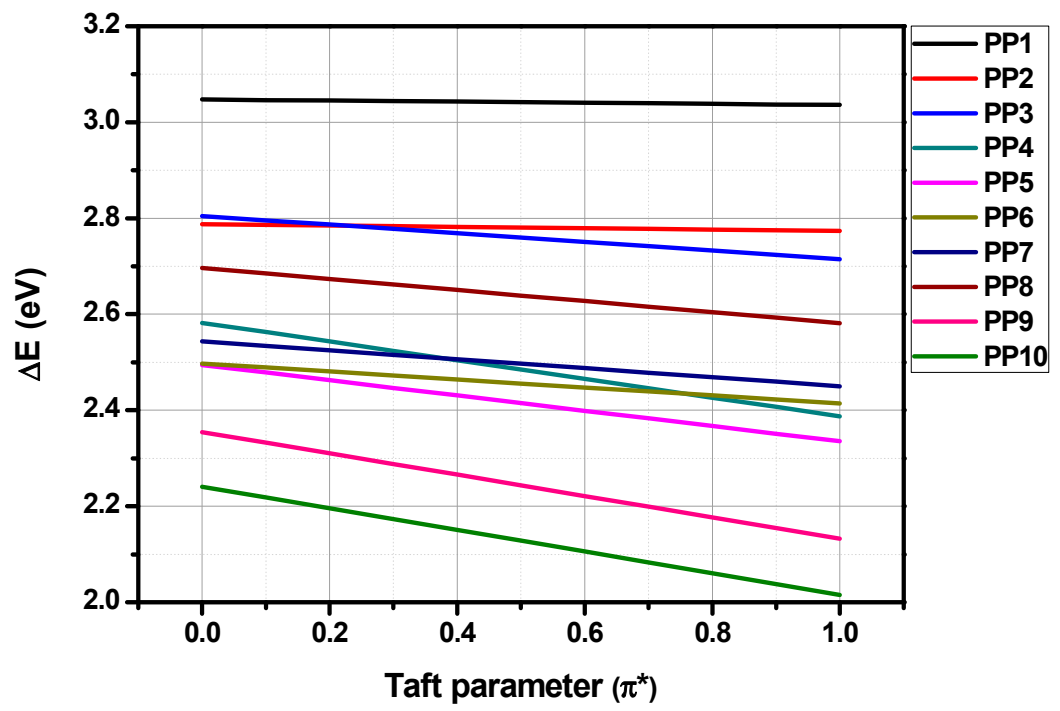

(a)

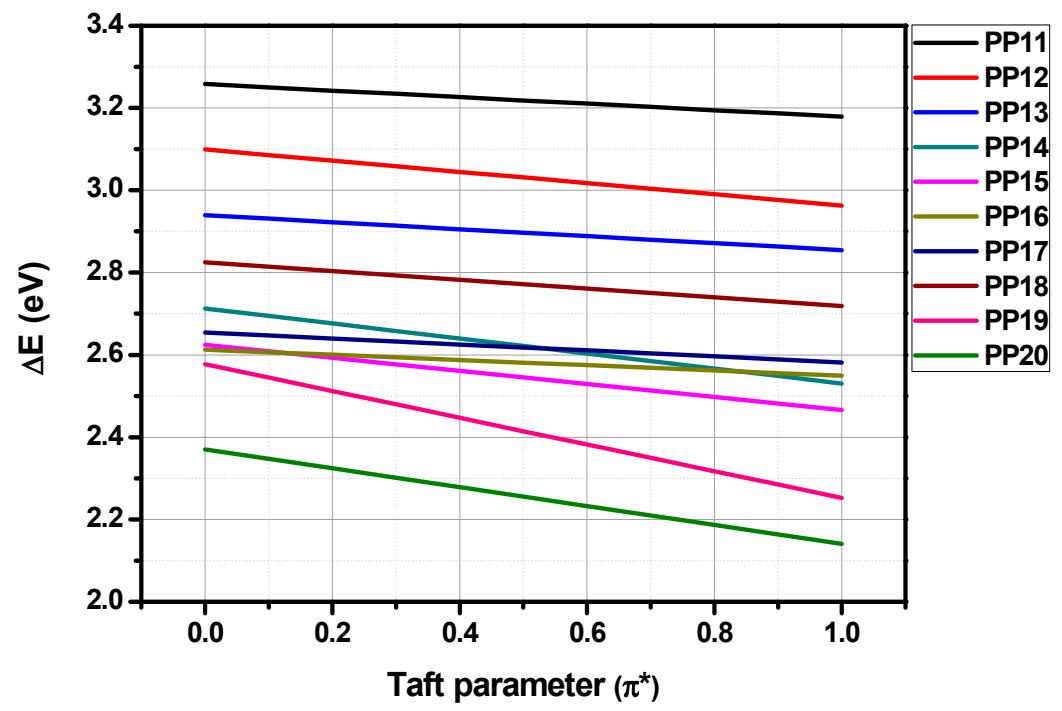

(b)

Figure 9. Variation of the positions of the charge transfer band with Kamlet-Taft empirical parameters for PP1-PP10 (a) and PP11-PP20 (b).

\subsection{Fluorescence Spectroscopy}

The fluorescent properties of all compounds were investigated in dichloromethane and toluene, in diluted solutions and the results are summarized in the Figure 10 and the Table 5. Interestingly, most of the chromophores were not emissive whatever the solvent is and this behavior is consistent with results classically reported in the literature for indane-1,3-dione derivatives [57-61]. However, it has to be noticed that a series of indane-1,3-dione derivatives was reported as being highly emissive, in a specific context, by use of oligo(phenylene)vinylene as electron donors [62]. Only eleven of these compounds displayed a weak emission in toluene so that the luminescence lifetime as well as the 
photoluminescence quantum yield were not determined for these compounds. While examining the emission maxima, the most red-shifted emissions were found for PP9 and PP10 displaying the most extended conjugated spacer but also the most extended electron acceptor. As attended, a blue-shifted emission was found for PP20 $\left(\lambda_{\mathrm{em}}=604 \mathrm{~nm}\right)$ relative to that of PP10 $\left(\lambda_{\mathrm{em}}=626 \mathrm{~nm}\right)$, directly resulting from its blue-shifted absorption maxima. While comparing the results obtained in dichloromethane and toluene, absorption maxima were found to be blue-shifted by $c a .20 \mathrm{~nm}$ for all dyes in toluene relative to that determined in dichloromethane. Based on the Taft parameters for both solvents ( 0.54 for toluene and 0.82 for dichloromethane), it can be concluded that a positive solvatochromism can also be observed in emission. This point is notably confirmed by the Stokes shifts determined in both dichloromethane and toluene, which are almost identical.

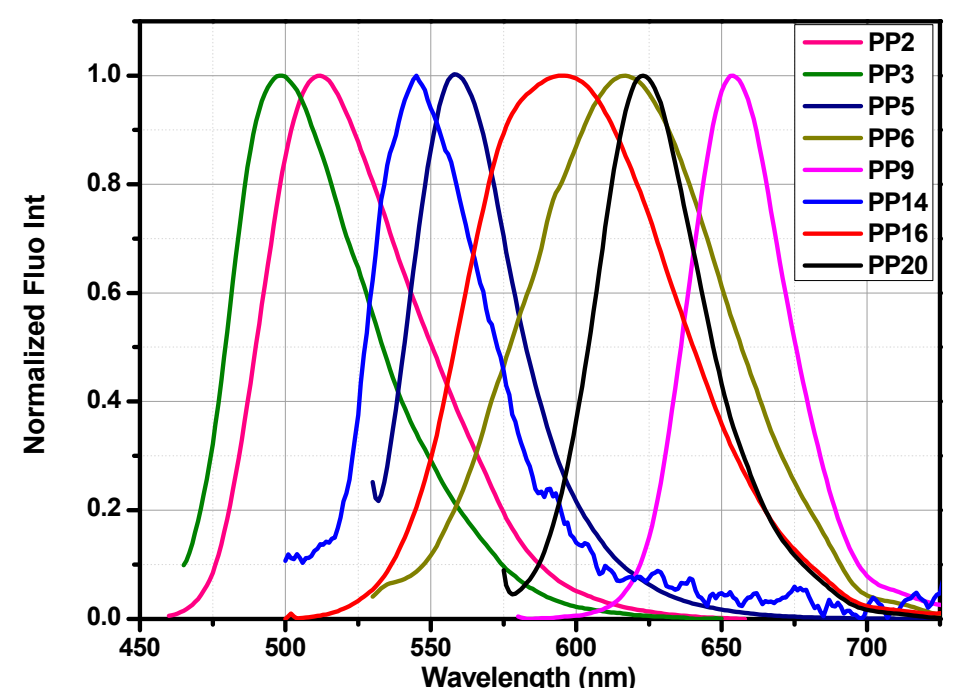

(a)

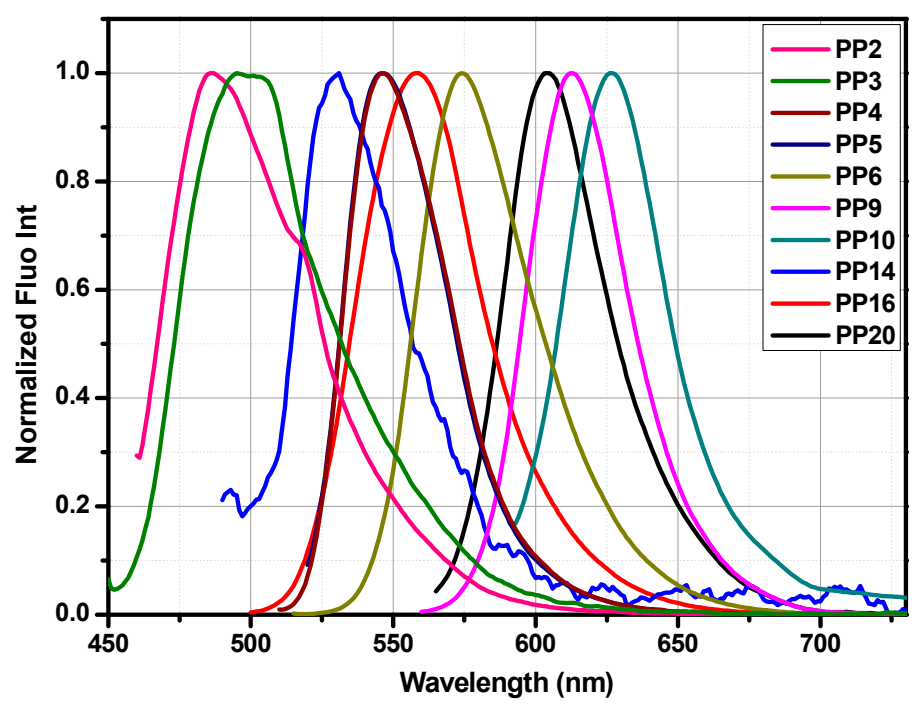

(b)

Figure 10. Fluorescence spectra of studied compounds in dichloromethane (a) and toluene (b). 
Table 5. Fluorescence properties of the different compounds in dichloromethane and toluene solutions.

\begin{tabular}{|c|c|c|c|c|c|c|c|c|c|c|}
\hline \multicolumn{11}{|c|}{ Dichloromethane } \\
\hline Compounds & PP1 & PP2 & PP3 & PP4 & PP5 & PP6 & PP7 & PP8 & PP9 & PP10 \\
\hline excitation (nm) & 411 & 448 & 456 & 510 & 524 & 524 & 505 & 476 & 571 & 603 \\
\hline emission (nm) & - & 512 & 499 & - & 558 & 617 & - & - & 654 & - \\
\hline Stokes shift (nm) & - & 64 & 43 & - & 34 & 103 & - & - & - & - \\
\hline Compounds & PP11 & PP12 & PP13 & PP14 & PP15 & PP16 & PP17 & PP18 & PP19 & PP20 \\
\hline excitation (nm) & 388 & 417 & 433 & 482 & 497 & 489 & 482 & 452 & 537 & 567 \\
\hline emission (nm) & - & - & - & 545 & - & 596 & - & - & - & 623 \\
\hline Stokes shift (nm) & - & - & - & 63 & - & 107 & - & - & - & 56 \\
\hline \multicolumn{11}{|c|}{ Toluene } \\
\hline Compounds & PP1 & PP2 & PP3 & PP4 & PP5 & PP6 & PP7 & PP8 & PP9 & PP10 \\
\hline excitation $(\mathrm{nm})$ & 405 & 450 & 441 & 501 & 512 & 509 & 501 & 473 & 553 & 582 \\
\hline emission (nm) & - & 487 & 495 & 546 & 546 & 574 & - & - & 613 & 626 \\
\hline Stokes shift (nm) & - & 37 & 54 & 45 & 34 & 65 & - & - & 60 & 44 \\
\hline Compounds & PP11 & PP12 & PP13 & PP14 & PP15 & PP16 & PP17 & PP18 & PP19 & PP20 \\
\hline excitation (nm) & 388 & 408 & 429 & 474 & 486 & 485 & 476 & 451 & 520 & 552 \\
\hline emission (nm) & - & 467 & - & 530 & - & 558 & - & - & - & 604 \\
\hline Stokes shift (nm) & - & 59 & - & 56 & - & 73 & - & - & - & 52 \\
\hline
\end{tabular}

\subsection{Electrochemical Properties}

The electrochemical properties of all compounds have been investigated by cyclic voltammetry $(\mathrm{CV})$ in dilute solutions, either in acetonitrile or in dichloromethane. The selected voltammograms are shown in the Figure 11 and CV curves of all compounds are given in the Supplementary Materials. The redox potentials of all compounds are summarized in the Table 6 in which redox potentials are given against the half wave oxidation potential of the ferrocene/ferrocenium cation couple.

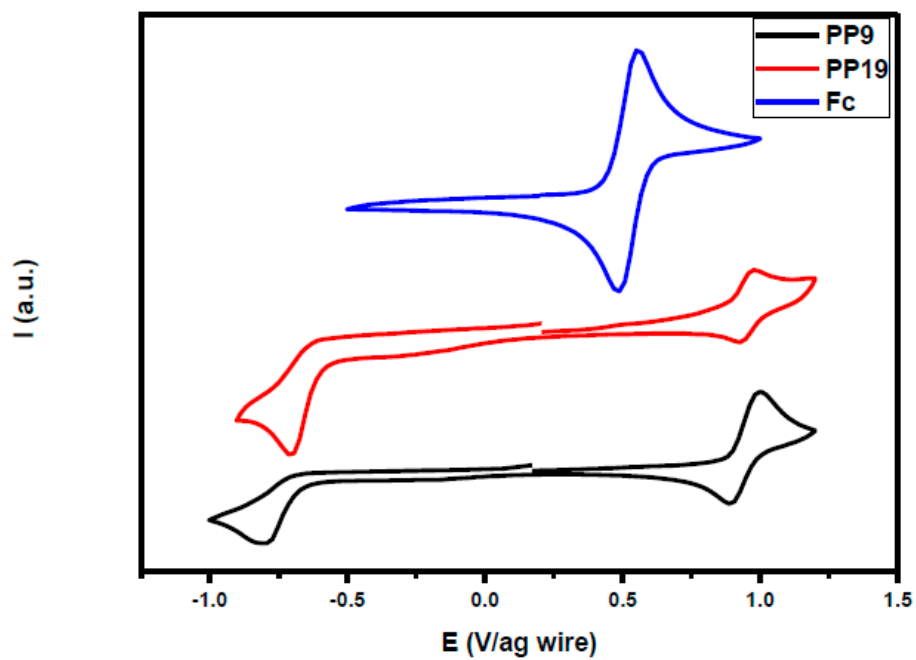

Figure 11. Selected examples of cyclic voltammograms of studied compounds (PP9, PP19) and Ferrocene $\left(\mathrm{Fc}_{\mathrm{C}}\right)$.

Table 6. Electrochemical redox potentials of the studied compounds PP1-PP20.

\begin{tabular}{ccccccc}
\hline Compounds & $\mathrm{E}_{\text {red }}(\mathrm{V} / \mathrm{Fc})$ & $\mathrm{E}_{\mathbf{O x}}(\mathrm{V} / \mathrm{Fc})$ & $\mathrm{E}_{\text {HOMO }}(\mathrm{eV})$ & $\mathrm{E}_{\text {LUMO }}(\mathrm{eV})$ & $\Delta \mathrm{E}_{\mathrm{el}}(\mathrm{eV})^{\mathbf{1}}$ & $\Delta \mathrm{E}_{\mathbf{o p t}}(\mathrm{eV})^{\mathbf{2}}$ \\
\hline PP1 & -1.30 & 1.19 & -5.99 & -3.50 & 2.49 & 3.07 \\
PP2 & -1.26 & 1.18 & -5.98 & -3.54 & 2.44 & 2.82 \\
PP3 & -1.36 & 1.15 & -5.95 & -3.44 & 2.51 & 2.76 \\
\hline
\end{tabular}


Table 6. Cont.

\begin{tabular}{|c|c|c|c|c|c|c|}
\hline Compounds & $E_{\text {red }}(V / F c)$ & $E_{O x}(V / F c)$ & $\mathrm{E}_{\text {HOMO }}(\mathrm{eV})$ & $\mathrm{E}_{\text {LUMO }}(\mathrm{eV})$ & $\Delta \mathrm{E}_{\mathrm{el}}(\mathrm{eV})^{1}$ & $\Delta \mathrm{E}_{\mathrm{opt}}(\mathrm{eV})^{2}$ \\
\hline PP4 & -1.33 & 0.68 & -5.48 & -3.47 & 2.01 & 2.45 \\
\hline PP5 & -1.40 & 0.63 & -5.43 & -3.40 & 2.03 & 2.37 \\
\hline PP6 & -1.32 & 0.79 & -5.59 & -3.48 & 2.11 & 2.46 \\
\hline PP7 & -1.26 & 0.84 & -5.64 & -3.54 & 2.10 & 2.50 \\
\hline PP8 & -1.38 & 0.95 & -5.75 & -3.42 & 2.33 & 2.63 \\
\hline PP9 & -1.22 & 0.49 & -5.29 & -3.58 & 1.71 & 2.19 \\
\hline PP10 & -1.30 & 0.41 & -5.21 & -3.50 & 1.71 & 2.08 \\
\hline PP11 & -1.46 & 1.19 & -5.99 & -3.34 & 2.65 & 3.24 \\
\hline PP12 & -1.44 & 1.18 & -5.98 & -3.36 & 2.62 & 3.02 \\
\hline PP13 & -1.50 & 1.23 & -6.03 & -3.30 & 2.73 & 2.90 \\
\hline PP14 & -1.56 & 0.65 & -5.45 & -3.24 & 2.21 & 2.58 \\
\hline PP15 & -1.30 & 0.54 & -5.34 & -3.50 & 1.84 & 2.51 \\
\hline PP16 & -1.44 & 0.77 & -5.57 & -3.36 & 2.21 & 2.60 \\
\hline PP17 & -1.29 & 0.85 & -5.65 & -3.51 & 2.14 & 2.63 \\
\hline PP18 & -1.52 & 0.94 & -5.74 & -3.28 & 2.46 & 2.78 \\
\hline PP19 & -1.30 & 0.49 & -5.29 & -3.50 & 1.79 & 2.35 \\
\hline PP20 & -1.30 & 0.36 & -5.16 & -3.50 & 1.66 & 2.23 \\
\hline
\end{tabular}

${ }^{1}$ All potentials are recorded in $0.1 \mathrm{M} \mathrm{TBABF}_{4} / \mathrm{CH}_{3} \mathrm{CN}$, except for PP15 and PP20 for which electrochemistry was carried out in $0.1 \mathrm{M} \mathrm{TBAClO}_{4} / \mathrm{CH}_{2} \mathrm{Cl}_{2}$. $\mathrm{E}_{\mathrm{HOMO}}(\mathrm{eV})=-4.8-\mathrm{E}_{\mathrm{Ox}}$ and $\mathrm{E}_{\mathrm{LUMO}}(\mathrm{eV})=-4.8-\mathrm{E}_{\text {red }}{ }^{2}$ Optical bandgaps determined in acetonitrile.

As shown in Figure 11, PP9 and PP19 differ from each other only in the nature of the accepting moiety. As expected, both compounds have quasi-reversible oxidation processes with identical oxidation potentials (Figure 11, Table 6). Indeed, the oxidation process for the two chromophores is centered onto the electron-donating part and this latter is the same for the two dyes. Conversely, the reduction potential of PP9 comprising $1 H$-cyclopenta[ $b]$ naphthalene-1,3(2H)-dione (EA4) as the electron-accepting moiety is slightly lower than that of PP19 (comprising EA1 as the acceptor), leading to narrower electrochemical bandgap. This is in good accordance with the optical bandgap determined by UV-visible absorption spectroscopy, where PP9 showed a red-shifted ICT band in comparison to PP19 (See Figures 7 and 8).

The redox potentials of all other compounds are gathered in the Table 6. As can be seen in the same series (PP1-PP10 and PP11-PP20) where the nature of the acceptor moiety is identical, their reduction potentials changed very slightly while the oxidation potential importantly vary as a function of the donor moiety.

The number and the substitution position of the alkoxy chains on the phenyl ring slightly influence the redox potentials of the targeted molecules (PP1-3 and PP11-13). However, important variations were determined when the electron-donating ability of the electron donor was increased by the presence of diakylamino groups on the phenyl ring (see PP4-5 and PP14-15). This phenomenon was even much more pronounced when a double bond was inserted between the donor and the acceptor moiety leading to more conjugated push pull molecules (see PP9-10 \& PP19-20). The presence of two 4-(N,N-methylamino)phenyl groups such as in PP10 and PP20 has only a negligible impact on the electrochemical property. In fact, while the second $4-(N, N$-methylamino)phenyl group could increase the electron donating ability, examination of the mesomeric forms in PP10 and PP20 clearly evidences the two groups not to be able to contribute simultaneously to the electronic delocalization, as previously mentioned in the literature [46]. While comparing the dyes at identical electron donating groups, push pull compounds prepared with EA4 (PP1-PP10) have lower reduction potentials than their counterparts PP11-PP20 comprising EA1 as the electron withdrawing groups. This is in perfect accordance with the higher electron accepting capacity of EA4.

The redox behaviors of synthesized molecules were then used to estimate their HOMO and LUMO energy levels by using the ferrocene $(\mathrm{Fc})$ ionization potential value ( $4.8 \mathrm{eV}$ vs. vacuum) as the standard. The correcting factor of $4.8 \mathrm{eV}$ is based on calculations obtained by Pommerehne et al. [63]. It is also 
important to note that some other correcting factors have also been used in the literature [64]. The obtained values of $\mathrm{E}_{\mathrm{HOMO}}$ and $\mathrm{E}_{\mathrm{LUMO}}$ issued from electrochemical characterizations are summarized in the Table 6 for comparison, the optical bandgaps of all dyes in acetonitrile have been added. The Figure 12 shows a comparative presentation of the frontier orbitals' energy levels experimentally and theoretically obtained. We can see that the experimental findings fit well with the trend predicted by DFT calculation.

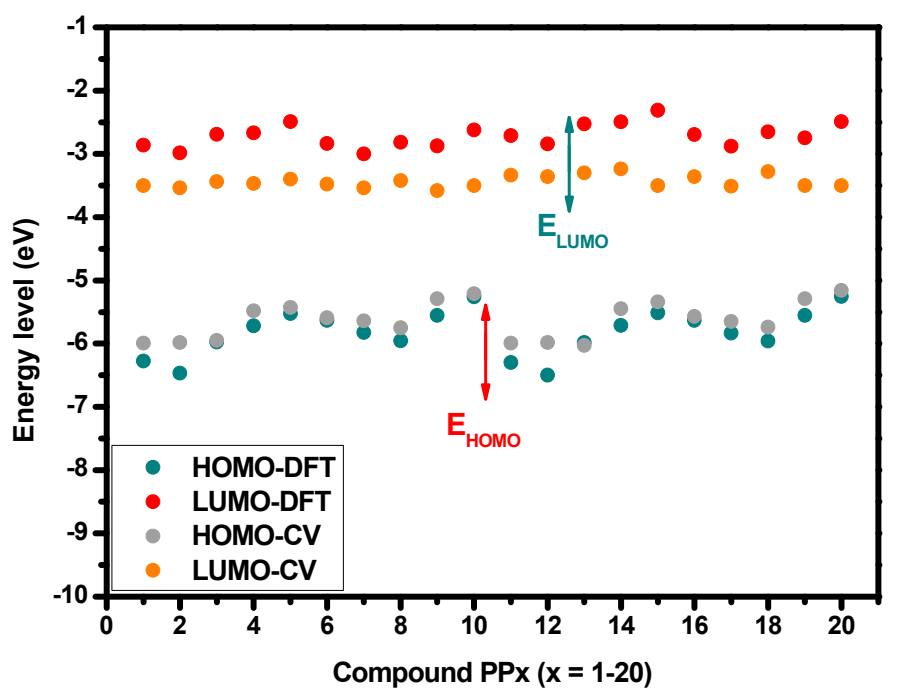

Figure 12. Comparison of frontier orbitals' energy levels obtained from cyclic voltammetry and DFT calculations.

\section{Conclusions}

In this work, a series of twenty dyes comprising indane-1,3-dione or $1 H$-cyclopenta[b] naphthalene-1,3(2H)-dione have been synthesized and examined for their photophysical properties. Introduction of a naphthalene moiety in EA4 greatly contributed to improve the electron-withdrawing ability of the group. Notably, a red-shift of the intramolecular charge transfer band of ca. $30 \mathrm{~nm}$ was observed for all dyes prepared with this acceptor, compared to the parent series comprising EA1. A linear and positive solvatochromism was found for all dyes, demonstrating an important charge redistribution upon excitation. A good correlation between the experimental HOMO-LUMO gaps and the theoretical ones could be found. Examination of their electrochemical properties revealed these dyes to be reversibly oxidized whereas an irreversible reduction monoelectronic process was determined for all dyes. By extending the aromaticity of EA4, a significant red-shift of the absorption maximum could be obtained for all dyes compared their analogues comprising EA1. Future works will consist of further improving the electron accepting ability of EA4, which is achievable by functionalizing EA4 with malononitrile. Based on the present work, the tetracyano derivatives of EA4 will certainly allow the design of near-infrared dyes that can find applications in research fields such as telecommunications and defense.

Supplementary Materials: The following are available online at http://www.mdpi.com/1996-1944/12/8/1342/s1. ${ }^{1} \mathrm{H}$ and ${ }^{13} \mathrm{C} \mathrm{nmR}$ spectra of all chromophores; Solvatochromism analyses in 23 solvents; contour plots of HOMO and LUMO energy levels of all dyes; cyclic voltammograms of all chromophores.

Author Contributions: C.P.; G.N. and F.D. directed the work and supervised the overall project. C.P., G.N. and F.D. carried out the synthesis of the chromophores, the chemical characterization of the dyes, the UV-visible absorption measurements and the solvatochromism experiments. T.-T.B. carried out the fluorescence and cyclic voltammetry experiments. S.P. carried out the theoretical calculations. Writing-Original Draft Preparation, C.P., G.N., F.D., T.-T.B. and S.P.; Writing-Review \& Editing, C.P., G.N. and F.D.; M.N. and D.G. contributed to review the manuscript. All authors approved the final manuscript. 
Funding: This research was funded by Aix Marseille University and The Centre National de la Recherche (CNRS) for financial supports. This research was also funded by the Agence Nationale de la Recherche (ANR agency) through the PhD grants of Corentin Pigot (ANR-17-CE08-0010 DUALITY project) and Guillaume Noirbent (ANR-17-CE08-0054 VISICAT project).

Conflicts of Interest: The authors declare no conflict of interest.

\section{References}

1. Raimundo, J.M.; Blanchard, P.; Gallego-Planas, N.; Mercier, N.; Ledoux-Rak, I.; Hierle, R.; Roncali, J. Design and synthesis of push-pull chromophores for second-order nonlinear optics derived from rigidified thiophene-based $\pi$-conjugating spacers. J. Org. Chem. 2002, 67, 205-218. [CrossRef]

2. El-Shishtawy, R.M.; Borbone, F.; Al-amshany, Z.M.; Tuzi, A.; Barsella, A.; Asiri, A.M.; Roviello, A. Thiazole azo dyes with lateral donor branch: Synthesis, structure and second order NLO properties. Dyes Pig. 2013, 96, 45-51. [CrossRef]

3. Paek, S.; Lee, J.K.; Ko, J. Synthesis and photovoltaic characteristics of push-pull organic semiconductors containing an electron-rich dithienosilole bridge for solution-processed small-molecule organic solar cells. Sol. Energ. Mater. Solar Cells 2014, 120, 209-217. [CrossRef]

4. Xu, S.J.; Zhou, Z.; Liu, W.; Zhang, Z.; Liu, F.; Yan, H.; Zhu, X. A twisted thieno[3,4-b]thiophene-based electron acceptor featuring a $14-\pi$-electron indenoindene core for high-performance organic photovoltaics. Adv. Mater. 2017, 29, 1704510. [CrossRef]

5. Karak, S.; Liu, F.; Russell, T.P.; Duzhko, V.V. Bulk charge carrier transport in push-pull type organic semiconductor. ACS Appl. Mater. Interfaces 2014, 6, 20904-20912. [CrossRef] [PubMed]

6. Turkoglu, G.; Cinar, M.E.; Ozturk, T. Triarylborane-based materials for OLED applications. Molecules 2017, 22, 1522. [CrossRef] [PubMed]

7. Archetti, G.; Abbotto, A.; Wortmann, R. Effect of polarity and structural design on molecular photorefractive properties of heteroaromatic-based push-pull dyes. Chem. Eur. J. 2006, 12, 7151-7160. [CrossRef] [PubMed]

8. Ipuy, M.; Billon, C.; Micouin, G.; Samarut, J.; Andraud, C.; Bretonnière, Y. Fluorescent push-pull $\mathrm{pH}$-responsive probes for ratiometric detection of intracellular pH. Org. Biomol. Chem. 2014, 12, 3641-3648. [CrossRef] [PubMed]

9. Gao, S.-H.; Xie, M.-S.; Wang, H.-X.; Niu, H.-Y.; Qu, G.-R.; Guo, H.-M. Highly selective detection of $\mathrm{Hg}^{2+}$ ion by push-pull-type purine nucleoside-based fluorescent sensor. Tetrahedron 2014, 70, 4929-4933. [CrossRef]

10. Jones, R.M.; Lu, L.; Helgeson, R.; Bergstedt, T.S.; McBranch, D.W.; Whitten, D.G. Building highly sensitive dye assemblies for biosensing from molecular building blocks. Proc. Nat. Acad. Sci. USA 2001, 98, 14769-14772. [CrossRef]

11. Ziessel, R.; Ulrich, G.; Harriman, A.; Alamiry, M.A.H.; Stewart, B.; Retailleau, P. Solid-state gas sensors developed from functional difluoroboradiazaindacene dyes. Chem. Eur. J. 2009, 15, 1359-1369. [CrossRef]

12. Tehfe, M.-A.; Dumur, F.; Graff, B.; Gigmes, D.; Fouassier, J.-P.; Lalevée, J. Blue-to-red light sensitive push-pull structured photoinitiators: indanedione derivatives for radical and cationic photopolymerization reactions. Macromolecules 2013, 46, 3332-3341. [CrossRef]

13. Tehfe, M.-A.; Dumur, F.; Graff, B.; Morlet-Savary, F.; Gigmes, D.; Fouassier, J.-P.; Lalevée, J. New push-pull dyes derived from Michler's ketone for polymerization reactions upon visible lights. Macromolecules 2013, 46, 3761-3770. [CrossRef]

14. Tehfe, M.-A.; Dumur, F.; Graff, B.; Morlet-Savary, F.; Gigmes, D.; Fouassier, J.-P.; Lalevée, J. Push-pull (thio)barbituric acid derivatives in dye photosensitized radical and cationic polymerization reactions under 457/473 nm Laser beams or blue LEDs. Polym. Chem. 2013, 4, 3866-3875. [CrossRef]

15. Mokbel, H.; Telitel, S.; Dumur, F.; Vidal, L.; Versace, D.-L.; Tehfe, M.-A.; Graff, B.; Toufaily, J.; Fouassier, J.-P.; Gigmes, D.; Hamieh, T.; Lalevée, J. Photoinitiating systems of polymerization and in-situ incorporation of metal nanoparticles in polymer matrixes upon visible lights: push-pull malonate and malonitrile based dyes. Polym. Chem. 2013, 4, 5679-5687. [CrossRef]

16. Xiao, P.; Frigoli, M.; Dumur, F.; Graff, B.; Gigmes, D.; Fouassier, J.-P.; Lalevée, J. Julolidine or fluorenone based push-pull dyes for polymerization upon soft polychromatic visible light or green light. Macromolecules 2014, 47, 106-112. [CrossRef] 
17. Bures, F. Fundamental aspects of property tuning in push-pull molecules. RSC Adv. 2014, 4, 58826-58851. [CrossRef]

18. Noirbent, G.; Dumur, F. Recent advances on nitrofluorene derivatives: Versatile electron acceptors to create dyes absorbing from the visible to the near and far-infrared region. Materials 2018, 11, 2425. [CrossRef] [PubMed]

19. Solanke, P.; Ruzicka, A.; Mikysek, T.; Pytela, O.; Bures, F.; Klikar, M. From linear to T-shaped indan-1,3-dione push-pull molecules: A comparative study. Helv. Chim. Acta 2018, 101, e201800090. [CrossRef]

20. Solanke, P.; Bures, F.; Pytela, O.; Klikar, M.; Mikysek, T.; Mager, L.; Barsella, A.; Ruzicková, Z. T-shaped (donor- $\pi$-)2acceptor- $\pi$-donor push-pull systems based on indan-1,3-dione. Eur. J. Org. Chem. 2015, 5339-5349. [CrossRef]

21. Swager, T.M.; Gutierrez, G.D. Push-pull chromophores from indan-1,3-dione. Synfacts 2014, 10, 0035.

22. Durand, R.J.; Gauthier, S.; Achelle, S.; Groizard, T.; Kahlal, S.; Saillard, J.-Y.; Barsella, A.; Le Poul, N.; Le Guen, F.R. Push-pull D- $\pi-R u-\pi-A$ chromophores: synthesis and electrochemical, photophysical and second order nonlinear optical properties. Dalton Trans. 2018, 47, 3965-3975. [CrossRef]

23. Nishiyabu, R.; Anzenbacher, P. 1,3-Indane-based chromogenic calixpyrroles with push-pull chromophores: Synthesis and anion sensing. Org. Lett. 2006, 8, 359-362. [CrossRef] [PubMed]

24. Cui, Y.; Ren, H.; Yu, J.; Wang, Z.; Qian, G. An indanone-based alkoxysilane dye with second order nonlinear optical properties. Dyes Pigm. 2009, 81, 53-57. [CrossRef]

25. Batsanov, A.S.; Bryce, M.R.; Davies, S.R.; Howard, J.A.K.; Whitehead, R.; Tanner, B.K. Studies on $\pi$-acceptor molecules containing the dicyanomethylene group. X-ray crystal structure of the charge-transfer complex of tetramethyltetrathiafulvalene and 2,3-dicyano-1,4-naphthoquinone: (TMTTF) $)_{3}(\mathrm{DCNQ})_{2}$. J. Chem. Soc. Perkin Trans. 2 1993, 0, 313-319. [CrossRef]

26. Wadsworth, A.; Moser, M.; Marks, A.; Little, M.S.; Gasparini, N.; Brabec, C.J.; Baran, D.; McCulloch, I. Critical review of the molecular design progress in non-fullerene electron acceptors towards commercially viable organic solar cells. Chem. Soc. Rev. 2019. [CrossRef] [PubMed]

27. Liu, Y.; Li, M.; Zhou, X.; Jia, Q.-Q.; Feng, S.; Jiang, P.; Xu, X.; Ma, W.; Li, H.-B.; Bo, Z. Nonfullerene acceptors with enhanced solubility and ordered packing for high-efficiency polymer solar cells. ACS Energy Lett. 2018, 3, 1832-1839. [CrossRef]

28. Sanguinet, L.; Williams, J.C.; Yang, Z.; Twieg, R.J.; Mao, G.; Singer, K.D.; Wiggers, G.; Petschek, R.G. Synthesis and characterization of new truxenones for nonlinear optical applications. Chem. Mater. 2006, 18, 4259-4269. [CrossRef]

29. Sigalov, M.V.; Shainyan, B.A.; Chipanina, N.N.; Oznobikhina, L.P. Intra- and intermolecular hydrogen bonds in pyrrolylindandione derivatives and their interaction with fluoride and acetate: Possible anion sensing properties. J. Phys. Chem. A 2013, 117, 11346-11356. [CrossRef]

30. Feng, H.; Qiu, N.; Wang, X.; Wang, Y.; Kan, B.; Wan, X.; Zhang, M.; Xia, A.; Li, C.; Liu, F.; Zhang, H.; Chen, Y. An A-D-A type small-molecule electron acceptor with end-extended conjugation for high performance organic solar cells. Chem. Mater. 2017, 29, 7908-7917. [CrossRef]

31. Li, R.; Liu, G.; Xiao, M.; Yang, X.; Liu, X.; Wang, Z.; Ying, L.; Huang, F.; Cao, Y. Non-fullerene acceptors based on fused-ring oligomers for efficient polymer solar cells via complementary light-absorption. J. Mater. Chem. A 2017, 5, 23926-23936. [CrossRef]

32. Frisch, M.J.; Trucks, G.W.; Schlegel, H.B.; Scuseria, G.E.; Robb, M.A.; Cheeseman, J.R.; Montgomery, J.A., Jr.; Vreven, T.; Kudin, K.N.; Burant, J.C.; et al. GAUSSIAN Program; Gaussian, Inc.: Wallingford, CT, USA, 2009.

33. Lee, C.; Yang, W.; Parr, R.G. Development of the Colle-Salvetti correlation-energy formula into a functional of the electron density. Phys. Rev. B. Condens. Matter. 1988, 37, 785-789. [CrossRef]

34. Becke, A.D. A new mixing of Hartree-Fock and local density-functional theories. J. Chem. Phys. 1993, 98, 1372-1377. [CrossRef]

35. Hehre, W.J.; Ditchfield, R.; Pople, J.A. Self-consistent molecular orbital methods. XII. Further extensions of Gaussian-type basis sets for use in molecular orbital studies of organic molecules. J. Chem. Phys. 1972, 56, 2257-2261. [CrossRef]

36. Tomasi, J.; Mennucci, B.; Cances, E. The IEF version of the PCM solvation method: an overview of a new method addressed to study molecular solutes at the QM ab initio level. J. Mol. Struct. THEOCHEM 1999, 464, 211-226. [CrossRef] 
37. Scalmani, G.; Frisch, M.J. Continuous surface charge polarizable continuum models of solvation. I. General formalism. J. Chem. Phys. 2010, 132, 114110. [CrossRef] [PubMed]

38. O’Boyle, N.M.; Tenderholt, A.L.; Langner, K.M. cclib: A library for package-independent computational chemistry algorithms. J. Comp. Chem. 2008, 29, 839-845. [CrossRef]

39. Delong, W.; Lanying, W.; Yongling, W.; Shuang, S.; Juntao, F.; Xing, Z. Natural $\alpha$-methylenelactam analogues: Design, synthesis and evaluation of $\alpha$-alkenyl- $\gamma$ and $\delta$-lactams as potential antifungal agents against Colletotrichum orbiculare. Eur. J. Med. Chem. 2017, 130, 286-307. [CrossRef]

40. Zhang, Q.; Peng, H.; Zhang, G.; Lu, Q.; Chang, J.; Dong, Y.; Shi, X.; Wei, J. facile bottom-up synthesis of coronene-based 3-fold symmetrical and highly substituted nanographenes from simple aromatics. J. Am. Chem. Soc. 2014, 136, 5057-5064. [CrossRef] [PubMed]

41. Pollard, A.J.; Perkins, E.W.; Smith, N.A.; Saywell, A.; Goretzki, G.; Phillips, A.G.; Argent, S.P.; Sachdev, H.; Mueller, F.; Huefner, S.; et al. Supramolecular Assemblies Formed on an Epitaxial Graphene Superstructure. Angew. Chem. Int. Ed. 2010, 49, 1794-1799. [CrossRef]

42. He, F.; Tian, L.; Tian, X.; Xu, H.; Wang, Y.; Xie, W.; Hanif, M.; Xia, J.; Shen, F.; Yang, B.; et al. diphenylamine-substituted cruciform oligo(phenylene vinylene): enhanced one- and two-photon excited fluorescence in the solid state. Adv. Funct. Mater. 2007, 17, 1551-1557. [CrossRef]

43. Wang, H.; Ding, W.; Wang, G.; Pan, C.; Duan, M.; Yu, G. Tunable molecular weights of poly(triphenylamine-2,2'-bithiophene) and their effects on photovoltaic performance as sensitizers for dye-sensitized solar cells. J. Appl. Polym. Sci. 2016, 133, 44182. [CrossRef]

44. Al Mousawi, A.; Dumur, F.; Garra, P.; Toufaily, J.; Hamieh, T.; Graff, B.; Gigmes, D.; Fouassier, J.-P.; Lalevée, J. carbazole scaffold based photoinitiator/photoredox catalysts: toward new high performance photoinitiating systems and application in led projector 3d printing resins. Macromolecules 2017, 50, 2747-2758. [CrossRef]

45. Malina, I.; Kampars, V.; Turovska, B.; Belyakov, S. Novel green-yellow-orange-red light emitting donor- $\pi$-acceptor type dyes based on 1,3-indandione and dimedone moieties. Dyes Pigm. 2017, 139, 820-830. [CrossRef]

46. Dumur, F.; Mayer, C.R.; Dumas, E.; Miomandre, F.; Frigoli, M.; Sécheresse, F. New chelating stilbazonium-like dyes from michler's ketone. Org. Lett. 2008, 10, 321-324. [CrossRef]

47. Buckle, D.R.; Morgan, N.J.; Ross, J.W.; Smith, H.; Spicer, B.A. Antiallergic activity of 2-nitroindan-1, 3-diones. J. Med. Chem. 1973, 16, 1334-1339. [CrossRef]

48. Lee, Y.; Jo, A.; Park, S.B. Rational Improvement of Molar Absorptivity Guided by Oscillator Strength: A Case Study with Furoindolizine-Based Core Skeleton. Angew. Chem. 2015, 127, 15915-15919. [CrossRef]

49. Kamlet, M.J.; Abboud, J.-L.M.; Abraham, M.H.; Taft, R.W. Linear solvation energy relationships. 23. A comprehensive collection of the solvatochromic parameters, .pi.*, .alpha., and .beta., and some methods for simplifying the generalized solvatochromic equation. J. Org. Chem. 1983, 48, 2877-2887. [CrossRef]

50. Reichardt, C. Solvatochromic Dyes as Solvent Polarity Indicators. Chem. Rev. 1994, 94, 2319-2358. [CrossRef]

51. Lippert, E.Z. Dipolmoment und Elektronenstruktur von angeregten Molekülen. Naturforsch. 1955, 10a, 541-545. [CrossRef]

52. Catalan, J. On the ET (30), $\pi^{*}, \mathrm{Py}, \mathrm{S}^{\prime}$, and SPP empirical scales as descriptors of nonspecific solvent effects. J. Org. Chem. 1997, 62, 8231-8234. [CrossRef]

53. Kawski, A. Zur Lösungsmittelabhängigkeit der Wellenzahl von Elektronenbanden Lumineszierender Moleküle und über die Bestimmung der Elektrischen Dipolmomente im Anregungszustand. Acta Phys. Polon. 1966, 29, 507-518.

54. McRae, E.G. Theory of Solvent Effects on Molecular Electronic Spectra. Frequency Shifts. J. Phys. Chem. 1957, 61, 562-572. [CrossRef]

55. Suppan, P. Solvent effects on the energy of electronic transitions: experimental observations and applications to structural problems of excited molecules. J. Chem. Soc. A 1968, 0, 3125-3133. [CrossRef]

56. Bakshiev, N.G. Universal intermolecular interactions and their effect on the position of the electronic spectra of molecules in two component solutions. Opt. Spektrosk. 1964, 16, 821-832.

57. Redoa, S.; Eucat, G.; Ipuy, M.; Jeanneau, E.; Gautier-Luneau, I.; Ibanez, A.; Andraud, C.; Bretonnière, Y. Tuning the solid-state emission of small push-pull dipolar dyes to the far-red through variation of the electron-acceptor group. Dyes Pigm. 2018, 156, 116-132. 
58. Jeong, H.; Chitumalla, R.K.; Kim, D.W.; Prabhakar Vattikuti, S.V.; Thogiti, S.; Cheruku, R.; Kim, J.H.; Jang, J.; Koyyada, G.; Jung, J.H. The comparative study of new carboxylated 1,3-indanedione sensitizers with standard cyanoacetic acid dyes using co-adsorbents in dye-sensitized solar cells. Chem. Phys. Lett. 2019, 715, 84-90. [CrossRef]

59. Liu, Y.; Sun, Y.; Li, M.; Feng, H.; Ni, W.; Zhang, H.; Wan, X.; Chen, Y. Efficient carbazole-based small-molecule organic solar cells with an improved fill factor. RSC Adv. 2018, 8, 4867-4871. [CrossRef]

60. Cao, D.; Peng, J.; Hong, Y.; Fang, X.; Wang, L.; Meier, H. Enhanced performance of the dye-sensitized solar cells with phenothiazine-based dyes containing double D-A branches. Org. Lett. 2011, 13, 1610-1613. [CrossRef] [PubMed]

61. Dai, S.; Zhao, F.; Zhang, Q.; Lau, T.-K.; Li, T.; Liu, K.; Ling, Q.; Wang, C.; Lu, X.; You, W.; Zhan, X. Fused nonacyclic electron acceptors for efficient polymer solar cells. J. Am. Chem. Soc. 2017, 139, 1336-1343. [CrossRef]

62. Guerlin, A.; Dumur, F.; Dumas, E.; Miomandre, F.; Wantz, G.; Mayer, C.R. Tunable optical properties of chromophores derived from oligo(para-phenylene vinylene). Org. Lett. 2010, 12, 2382-2385. [CrossRef]

63. Pommerehne, J.; Vestweber, H.; Guss, W.; Mahrt, R.F.; Bässler, H.; Porsch, M.; Daub, J. Efficient two layer leds on a polymer blend basis. Adv. Mater. 1995, 7, 551-554. [CrossRef]

64. Cardona, C.M.; Li, W.; Kaifer, A.E.; Stockdale, D.; Bazan, G.C. Electrochemical considerations for determining absolute frontier orbital energy levels of conjugated polymers for solar cell applications. Adv. Mater. 2011, 23, 2367-2371. [CrossRef] [PubMed]

(C) 2019 by the authors. Licensee MDPI, Basel, Switzerland. This article is an open access article distributed under the terms and conditions of the Creative Commons Attribution (CC BY) license (http://creativecommons.org/licenses/by/4.0/). 